\title{
Productivité en biomasse du chêne liège dans une séquence de dégradation de la suberaie à Cytise de Kroumirie (Tunisie)
}

\author{
Houcine SEBEI $^{\mathrm{a} *}$, Ali AlBOUCHI ${ }^{\mathrm{b}}$, Maurice RAPPc ${ }^{\mathrm{c}}$, Mohamed Hédi EL AOUNI ${ }^{\mathrm{d}}$ \\ a École Supérieure d'Agriculture de Mograne, 1121 Mograne, Tunisie \\ b Institut National de Recherches en Génie Rural, Eaux et Forêts, BP 2, Ariana 2080, Tunisie \\ ${ }^{c}$ CNRS - CEFE L. Emberger, BP 5051, 34033 Montpellier, France \\ d Faculté des Sciences de Bizerte, Jarzouna 7021, Tunisie
}

(Received 7 November 2002; accepted 3 September 2003)

\begin{abstract}
Résumé - La productivité primaire nette $\left(\mathrm{PN}_{1}\right)$ des chênes lièges a été évaluée dans six parcelles plus ou moins dégradées de la suberaie à Cytise de Kroumirie, à partir des biomasses de douze arbres échantillons. Le comptage des cernes ligneux sur dix autres arbres, a permis de relier l'âge et l'accroissement radial du bois au diamètre à $1,30 \mathrm{~m}(\mathrm{DBH})$. Des relations allométriques ont été établies permettant d'estimer les productivités au niveau de l'arbre et de la parcelle. Au niveau de l'arbre, le DBH est une variable hautement explicative de l'accroissement en biomasse du liège de reproduction qui passe de 0,24 à $4,12 \mathrm{~kg} \mathrm{MS} \cdot \mathrm{arbre}^{-1} \cdot \mathrm{an}^{-1}$ pour des arbres de DBH variant entre 8,7 et $60 \mathrm{~cm}$. L'accroissement total en biomasse montre une forte augmentation avec l'âge de l'arbre passant de 2,1 à $14,6 \mathrm{~kg} \mathrm{MS} \cdot \mathrm{an}^{-1}$ entre 53 et 303 ans (DBH respectivement de 8,7 et $60 \mathrm{~cm}$ ) ; parallèlement, le taux de croissance relative (RGR) a chuté de 2,14 à $0,34 \% \cdot \mathrm{an}^{-1}$ et les contributions du liège de reproduction et du bois du tronc ont augmenté aux dépens des autres compartiments. Au niveau de la parcelle, la surface terrière, variable fortement liée au taux de recouvrement des arbres et très hautement explicative de $\mathrm{PN}_{1}$, est un indicateur quantitatif de dégradation. En effet, $\mathrm{PN}_{1} \mathrm{~s}$ 'élève à 5,2 $\mathrm{t}$ $\mathrm{MS} \cdot \mathrm{ha}^{-1} \cdot \mathrm{an}^{-1}\left(2341 \mathrm{kcal} \cdot \mathrm{m}^{-2} \cdot \mathrm{an}^{-1}\right)$ dans la parcelle la mieux conservée et à $0,7 \mathrm{t} \mathrm{MS} \cdot \mathrm{ha}^{-1} \cdot \mathrm{an}^{-1}\left(325 \mathrm{kcal} \cdot \mathrm{m}^{-2} \cdot \mathrm{an}^{-1}\right)$ au stade très dégradé. Les accroissements en biomasse des différents organes montrent également une baisse continue le long de cette séquence de dégradation passant respectivement de 1376 à 51 , de 892 à 70 et de 263 à $44 \mathrm{~kg} \mathrm{MS} \cdot \mathrm{ha}^{-1} \cdot \mathrm{an}^{-1}$, pour les troncs, les houppiers et les racines. Parallèlement, le liège de reproduction passe de 456 à $5 \mathrm{~kg} \mathrm{MS} \cdot \mathrm{ha}^{-1} \cdot \mathrm{an}^{-1}$ et les chutes totales de litière de 2,85 à $0,56 \mathrm{t} \mathrm{MS} \cdot \mathrm{ha}^{-1}$. $\mathrm{an}^{-1}$. Aux stades de dégradation avancée, on a enregistré une baisse sensible de l'allocation relative des assimilats au liège de reproduction, au bois du tronc et aux organes reproducteurs et au contraire une mobilisation des assimilats accrue par les rameaux et les racines.
\end{abstract}

productivité / suberaie / dégradation / surface terrière / Kroumirie

\begin{abstract}
Cork oak biomass productivity in a damaging sequence of cytisus cork oak forest in Kroumirie (Tunisia). The net primary productivity $\left(P N_{l}\right)$ of cork oaks was evaluated in six plots representing variable damage stages of cytisus cork oak forest in Kroumirie region using biomasses of twelve sampled trees. The counting of woody rings on an independent tree sample allowed to correlate the age and radial growth with the diameter at breast height $(\mathrm{DBH})$. Allometric relationships were established to estimate the productivities at individual and stand levels. At individual level, the DBH is a highly predictive parameter of reproduction cork; its production ranges from 0.24 to $4.12 \mathrm{~kg} \mathrm{DM}^{-\mathrm{Dree}^{-1}} \cdot \mathrm{yr}^{-1}$ for a DBH varying between 8.7 and $60 \mathrm{~cm}$. The total biomass increment sharply increased with tree age rising from 2.1 to $14.6 \mathrm{~kg} \mathrm{DM} \cdot \mathrm{tree}^{-1} \cdot \mathrm{yr}{ }^{-1}$ between 53 and 303 years $\left(8.7\right.$ and $60 \mathrm{~cm}$ of DBH). At the same time, the relative growth rate $(R G R)$ decreased from 2.14 to $0.34 \% \cdot \mathrm{yr}^{-1}$ and the contributions of reproduction cork and trunk wood increased at the expense of other compartments. At stand level, basal area, which is a variable closely related to the canopy covering and very highly predictive of $P N_{l}$, appears as a quantitative criterion of forest damaging. So, $P N_{l}$ reaches $5.2 \mathrm{t} \mathrm{DM} \cdot \mathrm{ha}^{-1} \cdot \mathrm{yr}^{-1}\left(2341 \mathrm{kcal} \cdot \mathrm{m}^{-2} \cdot \mathrm{yr}^{-1}\right)$ in the best preserved plot and only $0.7 \mathrm{t} \mathrm{DM} \cdot \mathrm{ha}^{-1} \cdot \mathrm{yr}^{-1}\left(325 \mathrm{kcal} \cdot \mathrm{m}^{-2} \cdot \mathrm{yr}^{-1}\right)$ in the most damaged one. The increments of the different compartments showed a continuous decrease along the whole damaging sequence, ranging from 1376 to 51,892 to 70 and 263 to $44 \mathrm{~kg} \mathrm{DM} \cdot \mathrm{ha}^{-1} \cdot \mathrm{yr}^{-1}$ for boles, crowns and roots, respectively. Simultaneously, reproduction cork decreased from 456 to $5 \mathrm{~kg} \mathrm{DM} \cdot \mathrm{ha}^{-1} \cdot \mathrm{yr}^{-1}$ and litterfall from 2.85 to $0.56 \mathrm{t} \mathrm{DM} \cdot \mathrm{ha}^{-1} \cdot \mathrm{yr}^{-1}$. At the advanced damaging stages, a significant decrease of the relative allocation of assimilates to reproduction cork, boles and reproductive organs occurred and inversely an increased mobilization of assimilates by the twigs and the roots.
\end{abstract}

productivity / cork oak forest / degradation / basal area / Kroumirie

\section{INTRODUCTION}

La suberaie tunisienne est en nette régression à l'image des suberaies du pourtour occidental méditerranéen [5, 42, 48, 59].
Cette régression a été attribuée aux cycles répétés de sécheresse $[4,19,57]$, à des attaques d'insectes phyllophages [4, 9, 21], à des incendies [42,59] et à la pression de l'homme et du cheptel par les coupes délictuelles, le défrichement et le surpâturage

\footnotetext{
* Corresponding author: sa.hou@planet.tn
} 
$[17,60]$. Cette situation s'est aggravée par l'absence de régénération naturelle issue de glands $[9,30,43]$ et d'opérations sylvicoles de protection et de renouvellement. Depuis 1896, date du début des premières exploitations de liège, une réglementation basée sur la méthode du jardinage ou de coupons réglés a été établie [1]. Dans la pratique, cette réglementation n'a été appliquée que pour l'opération de déliégeage (tous les 12 ans), les coupes de régénération et d'éclaircie prévues n'ayant pas été réalisées. En effet, l'Inventaire Forestier National [11] a montré que plus de la moitié des chênes lièges avait dépassé l'âge d'exploitabilité et nécessitait une coupe de rajeunissement. D'autre part, les plans d'aménagement de la suberaie tunisienne entrepris depuis 1965 ont été basés sur les estimations de récoltes de liège en appliquant un coefficient de déliégeage de 2 à 2,5 [47]. Actuellement, ces plans d'aménagement sont en cours de révision et devraient se baser sur la productivité primaire des chênes lièges selon l'état des peuplements. La rareté de ces données rend la tâche difficile car seul le liège de reproduction a fait l'objet d'évaluation de productivité au niveau de l'individu $[22,23,25,26]$ ou de la parcelle $[45,46]$. La productivité aérienne a été évaluée sur un seul peuplement de chênes lièges de Sicile [38] et aucune étude n'a porté sur la productivité souterraine ou sur l'impact de la dégradation. Par ailleurs, la productivité des chênes a été exprimée la plupart du temps en volume $[6,11,12]$. Or, la productivité en biomasse paraît, à notre avis, plus indiquée car elle tient compte de la densité et de l'humidité du bois et des caractéristiques de l'écorce, paramètres essentiels pour la comparaison des productivités entre les espèces.

Le présent travail se propose d'évaluer la productivité en biomasse des chênes lièges dans une séquence de dégradation de la suberaie à cytise de Kroumirie, caractérisée dans la présente étude par une diminution de la densité des chênes lièges, de leur surface terrière et de leur taux de recouvrement à l'hectare suite à la disparition des classes de forts diamètres [61]. Des relations de type allométrique ont été établies à partir des données dendrométriques et pondérales de douze arbres échantillons et ont permis d'évaluer les productivités totales et compartimentales au niveau de six parcelles. Les meilleures variables prédictives des productivités au niveau de l'arbre et de la parcelle et des indicateurs quantitatifs de la dégradation ont été identifiés.

\section{MATÉRIELS ET MÉTHODES}

\subsection{Stations d'étude}

La présente étude concerne six parcelles d'un hectare chacune, en fin de rotation, localisées dans trois stations de la suberaie à cytise (Quercus suber L. et Cytisus villosus Pourret) de Kroumirie, au nordouest de la Tunisie. Il s'agit des stations du col des Ruines (CR), Ben Métir (BM) et Ain Debba (AD) avec, dans chaque station, une parcelle en bon état (notée 1) et une autre plus ou moins dégradée (notée 2) [61]. Ces six parcelles forment une séquence de dégradation marquée par une baisse continue de la surface terrière des chênes lièges à l'hectare suite à la disparition des classes de forts diamètres, le stade 2 correspondant aux parcelles ayant une surface terrière inférieure à $14,8 \mathrm{~m}^{2} \cdot \mathrm{ha}^{-1}$ [61].

La productivité des chênes lièges dans les six parcelles d'étude repose sur les données dendrométriques et pondérales de douze arbres échantillons représentatifs de l'ensemble du peuplement : six dans la station (CR) et trois dans chacune des stations (BM) et (AD) [61]. Parmi ces douze arbres, neuf ayant subi au moins un démasclage auparavant ont fait l'objet de récolte de liège de reproduction, les trois autres étant encore trop jeunes.

\section{2. Âge des arbres et accroissement radial du bois}

Les âges de 10 autres arbres, abattus à l'occasion d'un élargissement de pare-feu à proximité de la station (AD), ont été déterminés par comptage des cernes annuels sur des rondelles prélevées à la base du tronc après séchage et polissage au papier abrasif. Des rondelles de tronc, prélevées sur ces mêmes arbres à 1,30 $\mathrm{m}$ de hauteur, ont servi au comptage des cernes en vue de calculer l'accroissement moyen annuel du bois à cette hauteur. Le nombre des cernes à la base et à $1,30 \mathrm{~m}$ a été corrélé au diamètre du bois à $1,30 \mathrm{~m}$ (ou DBH sous liège). La première équation obtenue a été utilisée dans le calcul de l'âge $(A)$ des 12 arbres échantillons et de l'âge moyen $\left(A_{i}\right)$ de chaque classe de diamètre $i$ et ceci à partir de leurs DBH respectifs. L'usage de cette relation peut être justifié car l'état des peuplements des parcelles (AD), jugé par la densité à l' hectare, le DBH moyen, la surface terrière et le recouvrement des chênes lièges, représente un stade intermédiaire dans la séquence de dégradation étudiée [61].

L'âge moyen $(\bar{A})$ du peuplement de chaque parcelle a été calculé selon la formule :

$$
\bar{A}=\frac{1}{N} \sum_{i=1}^{q} n_{i} A_{i}
$$

avec $N$ : effectif total de la parcelle ; $q$ : nombre total des classes de diamètre à $1,30 \mathrm{~m} ; A_{i}$ : âge moyen de la classe $i$ d'effectif $n_{i}$.

\subsection{Surface terrière et surface déliégée}

La surface terrière des chênes lièges de chaque parcelle a été calculée selon la formule :

$$
G\left(m^{2} \cdot h a^{-1}\right)=10^{-4} \sum_{i=1}^{q} n_{i} \pi\left[\frac{D_{i}^{2}(\mathrm{~cm})}{4}\right]
$$

avec $n_{i}$ : effectif de la classe de diamètre $i$ de DBH central $D_{i} ; q$ et $n_{i}$ sont les mêmes que ceux de la relation (1) puisqu'il s'agit des mêmes arbres.

La surface déliégée $(s d)$ des arbres échantillons ayant subi au moins un démasclage auparavant, a été calculée selon la formule :

$$
s d\left(m^{2} \cdot \text { arbre }^{-1}\right)=\pi \cdot D \cdot H d
$$

avec $: D=\mathrm{DBH}$ sous liège $(\mathrm{m}) ; H d=$ hauteur de déliégeage $(\mathrm{m})$; en assimilant la partie de la tige démasclée à un cylindre de diamètre $D$ et de hauteur $H d$.

Ces grandeurs ont été mises en relation avec le DBH des arbres échantillons et l'équation obtenue a été appliquée aux DBH centraux $D_{i}$ des différentes classes de diamètre pour évaluer la surface démasclée $s d i$ de l'arbre moyen de chaque classe. La surface déliégée par parcelle a été calculée selon la formule :

$$
S D\left(m^{2} \cdot h a^{-1}\right)=\sum_{i=1}^{q^{\prime}} n_{i} s d i
$$

avec $q^{\prime}$ : nombre de classes des arbres exploitables ayant un DBH sur liège supérieur à 12,8 cm [61]. 


\subsection{Taux de recouvrement des chênes lièges}

Le taux de recouvrement des chênes lièges dans chaque parcelle a été obtenu en appliquant à ses classes de DBH, une relation entre le $\mathrm{DBH}$ et la surface de projection du houppier, établie à partir de 69 arbres des parcelles étudiées [61].

\subsection{Productivité primaire du chêne liège}

La productivité primaire nette $\left(P N_{1}\right)$ comprend, essentiellement, l'accroissement de biomasse pérenne $(\Delta B)$, la chute de litière $(L)$ et la consommation de matière végétale par les phytophages $(C)[64,13]$. Seuls les deux premiers termes ont été évalués dans le présent travail.

Les biomasses épigée et hypogée des chênes lièges dans les six parcelles ont été calculées à l'aide de relations allométriques établies à partir des données dendrométriques et pondérales des arbres échantillons [61]. Pour la biomasse aérienne, neuf arbres dont le DBH sous liège était compris entre 2 et $23 \mathrm{~cm}$, avaient été abattus et séparés en différents compartiments; pour trois autres arbres de DBH compris entre 37 et $67,5 \mathrm{~cm}$ la biomasse sur pied avait été estimée à l'exception de celle du liège de reproduction obtenue après récolte. Ces estimations ont été basées sur des mesures de volume et de densité du liège et du bois en ce qui concerne les troncs et sur des relations allométriques tirées des données des arbres abattus dans le cas des houppiers. Les équations de régression établies entre les biomasses par compartiment des 12 arbres et leur DBH ont été appliquées aux classes de diamètre de chaque parcelle.

La biomasse des racines de toute taille a été établie à partir d'échantillons de sol prélevés sous 7 arbres abattus, à raison de deux prélèvements d'un quart de mètre carré chacun et de $40 \mathrm{~cm}$ de profondeur, effectués de part et d'autre de la souche, sans que celle-ci soit extraite [53]. Les biomasses racinaires obtenues ont été rapportées aux surfaces de projection du houppier des arbres. La relation établie entre la biomasse racinaire et le DBH de ces arbres échantillons a été appliquée aux classes de diamètre de chaque parcelle. Il faut signaler que cette technique ne tient pas compte du turn-over des racines fines au cours de l'année.

Dans les tarifs d'accroissement de la biomasse, nous avons distingué :

- le liège de reproduction, récolté sur une hauteur $H d$ tous les douze ans [1], dans le cas des arbres échantillons ayant subi au moins un démasclage auparavant ;

- le liège mâle du tronc situé au-dessus de $H d$ ou sur tout le tronc des très jeunes arbres. Dans le cas des trois arbres non abattus, il a été obtenu par différence entre le liège total estimé sur pied et le liège de reproduction ;

- les autres organes pérennes.

\subsubsection{Tarifs d'accroissement en biomasse au niveau de l'arbre}

Pour des parcelles en fin de rotation, la production annuelle de liège de reproduction des arbres échantillons (obtenue en divisant la biomasse de ce liège séché à l'étuve à $85^{\circ} \mathrm{C}$ par 12 , durée de la période de production du liège) a été corrélée avec leur DBH. Les biomasses des autres compartiments ont été mises en relation avec l'âge $(A)$ des 12 arbres échantillons. Ces relations ont servi à déterminer les accroissements en biomasse moyens par individu au cours des 12 dernières années, période de rotation du liège de reproduction. Pour cela, on suppose que les arbres échantillons constituent différents stades de développement d'un même arbre au cours du temps, les conditions des trois stations étudiées étant proches sur les plans climatique et édaphique. D'une part, elles appartiennent à un même bioclimat de type méditerranéen humide à variante hivernale tempérée ; d'autre part, leurs sols ont des niveaux de fertilité assez voisins. En effet, les teneurs moyennes des 30 premiers centimètres sont : 3 à $5 \%$ de matière organique, 0,12 à $0,15 \%$ d'azote total, 0,3 à $0,8 \%$ de $\mathrm{P}_{2} \mathrm{O}_{5}$ total et 5 à 6 ppm de $\mathrm{P}_{2} \mathrm{O}_{5}$ assimilable [61]

Le taux de croissance relatif ( $R G R$ ) d'un compartiment au cours d'une période $t$ s'écrit :

$$
R G R=\frac{\Delta b}{b m \cdot \Delta t}
$$

où $\frac{\Delta b}{\Delta t}$ représente l'accroissement en biomasse de ce compartiment et $b m$ sa biomasse moyenne dans l'intervalle $\Delta t$. Pour un très petit intervalle de temps $d t$, le taux de croissance s'écrit $\frac{1}{b} \frac{d b}{d t}$ qui peut être calculé de façon exacte comme $\frac{d(\log b)}{d t}$, soit :

$$
\frac{1}{d t}[\log b]_{t_{1}}^{t_{2}=t_{1}+d t}=\frac{1}{d t}\left(\log b_{2}-\log b_{1}\right)
$$

Pour un intervalle pas trop grand, ici 12 ans, le $R G R\left(\mathrm{en} \% \cdot \mathrm{an}^{-1}\right)$ peut alors s'écrire :

$$
R G R=\frac{100}{12}\left(\log b_{2}-\log b_{1}\right)
$$

où $b_{2}$ est la biomasse du compartiment de l'arbre au temps $t_{2}$ et $b_{1}$ sa biomasse au temps $t_{1}$ égal à $t_{2}-12$.

\subsubsection{Accroissement en biomasse au niveau de la parcelle}

Les accroissements moyens annuels de la biomasse au niveau de chaque parcelle durant les 12 dernières années, ont été obtenus en appliquant les tarifs individuels établis précédemment aux différentes classes de diamètre selon les formules suivantes :

- liège de reproduction $\left(\mathrm{en} \mathrm{kg} \cdot \mathrm{ha}^{-1} \cdot \mathrm{an}^{-1}\right)$ :

$$
\frac{\Delta B l}{\Delta t}=\sum_{i=1}^{q^{\prime}} n_{i} \frac{b l i}{12}
$$

où : $\frac{b l i}{12}\left(\mathrm{en} \mathrm{kg} \cdot \mathrm{an}^{-1}\right)$ est la production annuelle en liège de reproduction pour l'arbre moyen de la classe $i$, déduite du DBH central $D_{i}$ de la classe $i$

- feuilles et organes pérennes non exploités $\left(\mathrm{en} \mathrm{kg} \cdot \mathrm{ha}^{-1} \cdot \mathrm{an}^{-1}\right)$ :

$$
\frac{\Delta B}{\Delta t}=\frac{1}{12} \sum_{i=1}^{q^{\prime}} n_{i}\left[b_{i, t}-b_{i, t-12}\right]
$$

où $b_{i, t-12}$ et $b_{i, t}$ sont, respectivement, les biomasses par compartiment de l'arbre moyen de la classe $i$ en début (temps $t-12)$ et en fin (temps $t$ ) de rotation (en $\mathrm{kg}$ ) ;

- taux de croissance $R G R\left(\mathrm{en} \% \cdot \mathrm{an}^{-1}\right)$ de chaque compartiment:

$$
R G R=\frac{100}{12}\left(\log B_{2}-\log B_{1}\right)
$$

où $B_{2}$ et $B_{1}$ sont les biomasses du compartiment aux temps $t_{2}$ et $t_{2}-12$, respectivement.

L'équivalent énergétique des biomasses a été évalué en utilisant un coefficient de conversion moyen de $4,5 \mathrm{kcal} \cdot \mathrm{g}^{-1}$ de matière sèche [14].

Pour mettre en évidence l'influence de la dégradation, nous avons recherché les effets du recouvrement des chênes lièges et de leur surface terrière sur l'accroissement en biomasse des différents organes. En effet, ces deux paramètres dendrométriques de peuplement se sont avérés comme étant les meilleures variables explicatives des variations de la biomasse au niveau de la parcelle et des critères quantitatifs de l'état des peuplements [61].

\subsection{Chutes de litière}

Les litières de chêne liège ont été relevées à la fin de chaque mois durant deux ou trois années selon les parcelles entre 1987 et 1992. Les 
Table I. Paramètres dendrométriques et biomasses du tronc chez 12 chênes lièges de Kroumirie (Tunisie).

\begin{tabular}{|c|c|c|c|c|c|c|c|c|}
\hline \multirow{2}{*}{ Arbre } & \multirow{2}{*}{$\begin{array}{c}\text { DBH } \\
\text { Sous liège } \\
(\mathrm{cm})\end{array}$} & \multirow{2}{*}{$\begin{array}{c}\text { Âge } \\
\text { calculé } \\
\text { (ans) }\end{array}$} & \multirow{2}{*}{$\begin{array}{l}H d \\
(\mathrm{~m})\end{array}$} & \multirow{2}{*}{$\begin{array}{c}s d \\
\left(\mathrm{~m}^{2}\right)\end{array}$} & $\begin{array}{c}\text { Liège de } \\
\text { reproduction }\end{array}$ & Liège mâle & Liège total & Bois \\
\hline & & & & & \multicolumn{4}{|c|}{ (kgMS) } \\
\hline 1 & 2 & 14 & - & - & - & 0,623 & 0,623 & 0,712 \\
\hline 2 & 2 & 14 & - & - & - & 0,563 & 0,563 & 1,641 \\
\hline 3 & 3 & 20 & - & - & - & 0,945 & 0,945 & 1,142 \\
\hline 4 & 7 & 43 & 1,30 & 0,29 & 1,71 & 3,48 & 5,190 & 7,575 \\
\hline 5 & 8 & 49 & 1,41 & 0,35 & 14,65 & 4,058 & 18,708 & 28,297 \\
\hline 6 & 14 & 81 & 1,88 & 0,83 & 6,45 & 7,05 & 13,5 & 39,194 \\
\hline 7 & 17 & 97 & 1,73 & 0,92 & 6,726 & 9,724 & 16,45 & 57,982 \\
\hline 8 & 17 & 97 & 1,88 & 1,00 & 9,137 & 10,522 & 19,659 & 85,344 \\
\hline 9 & 23 & 127 & 2,36 & 1,71 & 15,518 & 17,622 & 33,14 & 84,127 \\
\hline 10 & 37 & 196 & 2,66 & 3,09 & 21,706 & 59,581 & 81,287 & 283,868 \\
\hline 11 & 54 & 275 & 2,82 & 4,78 & 36,071 & 62,076 & 98,147 & 521,182 \\
\hline 12 & 67,5 & 337 & 3,48 & 7,38 & 60,439 & 84,618 & 145,057 & 944,139 \\
\hline
\end{tabular}

$H d$ : hauteur de déliégeage ; $s d$ : surface déliégée.

retombées de litière de chaque parcelle ont été recueillies dans 8 bacs collecteurs disposés au hasard. Chaque bac, de forme carrée, avait une surface intérieure de $0,25 \mathrm{~m}^{2}$, une hauteur de $10 \mathrm{~cm}$ et disposait d'un fond de toile moustiquaire en matière plastique à mailles de $2 \mathrm{~mm}$; chaque bac était placé sur 4 pieds à $40 \mathrm{~cm}$ du sol [53]. Chaque récolte de litière a été ventilée en trois catégories : feuilles, bois mort et organes reproducteurs (inflorescences et glands).

\subsection{Calculs statistiques}

Les calculs de paramètres d'équations de régression ont été exécutés sur des variables non transformées à l'aide du logiciel « Microsoft Excel $2000 »$. Le coefficient de corrélation $(r)$ a été déterminé par analyse des données à l'aide de la fonction « corrélation » du même logiciel et son degré de signification a été vérifié par la « Table des valeurs critiques du coefficient de corrélation » [10]. L'analyse des résidus d'ajustement et le test de signification des coefficients de régression ont été effectués à l'aide du logiciel « SPSS 10,0 for Windows ». Le choix du modèle d'ajustement le mieux adapté aux données a été basé sur plusieurs critères : un meilleur coefficient de détermination $\left(R^{2}\right)$, des coefficients de régression significatifs au seuil de $95 \%$, une signification biologique du résultat, un écart type résiduel $(s)$ minimal et un meilleur coefficient de corrélation $\left(r^{\prime}\right)$ entre la valeur mesurée et la valeur prédite $[33,49]$

\section{RÉSULTATS}

\subsection{Au niveau de l'individu}

La figure 1 représente les relations entre le DBH sous liège des dix arbres abattus et l'âge total de l'arbre (A) d'une part, l'âge à 1,30 m (N) d'autre part. Ces deux équations, de type puissance ont des coefficients de corrélation très hautement significatifs :

$$
\begin{array}{ll}
\mathrm{A}=7,465 \mathrm{DBH}^{0,9044} & r=0,989 * * *(\alpha=0,001), \\
\mathrm{N}=6,122 \mathrm{DBH}^{0,9379} & r=0,988 * * *(\alpha=0,001) .
\end{array}
$$

La relation 10 suggère que l'exploitation du liège, portant théoriquement sur les arbres de DBH sous liège supérieur à $6,6 \mathrm{~cm}$ [61], débuterait à l'âge de 41 ans environ. Un arbre de taille moyenne $(20 \mathrm{~cm}$ de DBH) serait d'après la relation 10 âgé de 112 ans et aurait un accroissement radial ligneux de $1 \mathrm{~mm} \cdot \mathrm{an}^{-1}$

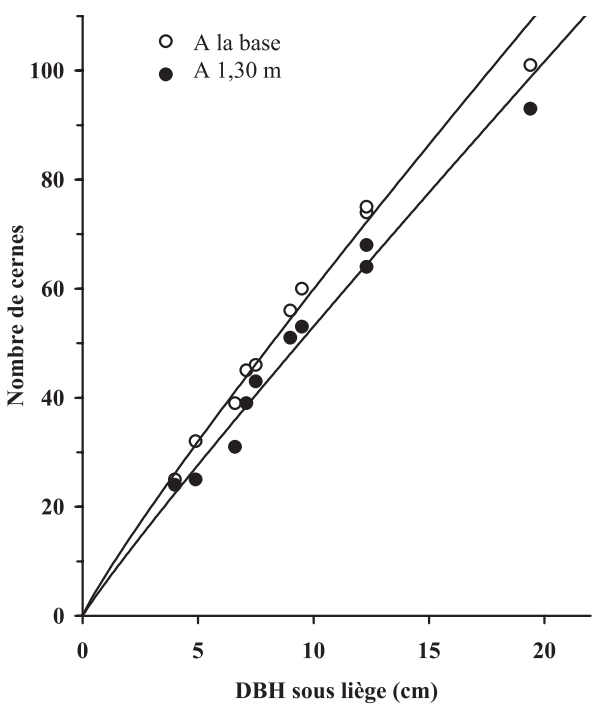

Figure 1. Relations entre le DBH sous liège, l'âge (A) et le nombre de cernes ligneux à $1,30 \mathrm{~m}(\mathrm{~N})$ pour un échantillon de dix chênes lièges de Kroumirie (Tunisie).

au cours de la dernière rotation (nombre de cernes à 1,30 m égal à 100).

Les données dendrométriques des 12 arbres échantillons et les biomasses des différents compartiments du tronc sont présentées au tableau I. La biomasse anormalement forte du liège de reproduction chez l'arbre 5 montre que cet arbre n'a pas été démasclé auparavant. Pour cela, il n'en a pas été tenu compte dans le tarif intermédiaire du liège de reproduction (bl) des arbres échantillons présenté à la figure $2 \mathrm{a}$ et très hautement corrélé au DBH par l'équation :

$\mathrm{bl}=0,0098 \mathrm{DBH}^{1,4756}$ avec $r=0,980 * * *(\alpha=0,001)$

Cette relation atteste que le DBH est une variable très hautement explicative de la production de liège au niveau de l'individu. Cette production, estimée à l'aide de la relation 12 , varie de 0,24 à $4,12 \mathrm{~kg} \mathrm{MS} \cdot$ arbre $^{-1} \cdot \mathrm{an}^{-1}$ pour un DBH compris entre $8,7 \mathrm{~cm}$ 

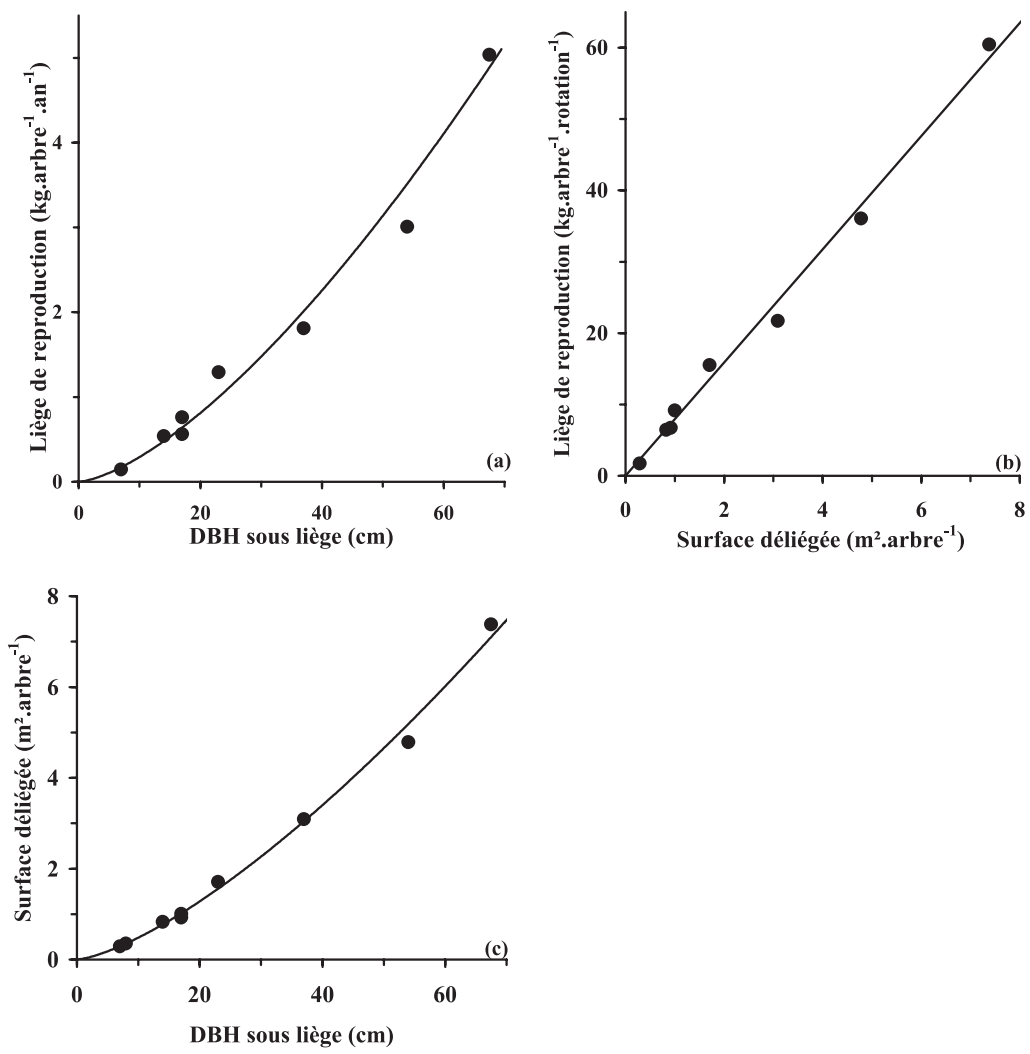

Figure 2. Relations entre la production du liège de reproduction et (a) le DBH sous liège (b) la surface déliégée et entre la surface déliégée et le DBH sous liège pour 8 arbres de l'échantillon étudié en Kroumirie (Tunisie).

Table II. Relations entre les biomasses des différents compartiments des chênes lièges ( $\mathrm{Y}$ en kg) et leur âge (A en ans).

\begin{tabular}{lcccccc}
\hline Compartiments & Modèle de régression & $n$ & $R^{2}$ & $s$ & $r^{\prime}$ & Relation \\
\hline Feuilles et brindilles & $\mathrm{Y}=0,0001 \mathrm{~A}^{2}+0,0547 \mathrm{~A}$ & 12 & 0,979 & 1,62 & 0,991 & $(15)$ \\
Rameaux & $\mathrm{Y}=0,0002 \mathrm{~A}^{2}+0,0479 \mathrm{~A}$ & 12 & 0,984 & 1,45 & 0,991 & $(16)$ \\
Bois des branches & $\mathrm{Y}=0,0046 \mathrm{~A}^{2}$ & 12 & 0,996 & 18,6 & 0,995 & $(17)$ \\
Liège des branches & $\mathrm{Y}=0,0025 \mathrm{~A}^{2}$ & 11 & 0,993 & 13,1 & 0,991 & $(18)$ \\
Bois du tronc & $\mathrm{Y}=0,0031 \mathrm{~A}^{2,162}$ & 12 & 0,976 & 0,39 & 0,996 & $(19)$ \\
Liège mâle du tronc & $\mathrm{Y}=0,0082 \mathrm{~A}^{1,594}$ & 11 & 0,989 & 0,19 & 0,972 & $(20)$ \\
Racines & $\mathrm{Y}=0,0014 \mathrm{~A}^{2}+0,1558 \mathrm{~A}$ & 6 & 0,940 & 4,40 & 0,972 & $(21)$ \\
\hline
\end{tabular}

$R^{2}$ : coefficient de détermination ; $s$ : écart type résiduel ; $r$ ': coefficient de corrélation entre Y mesurée et $\mathrm{Y}$ prédite.

(arbre âgé de 53 ans et soumis théoriquement à la première récolte de liège de reproduction) et $60 \mathrm{~cm}$. La surface déliégée (sdl) est également une variable très hautement explicative de la production de liège par individu (bl) comme le montrent la figure $2 \mathrm{~b}$ et son équation linéaire :

$$
\text { sdl }=7,9351 \mathrm{bl} \text { avec } r=0,996 * * *(\alpha=0,001)
$$

dont la pente exprime la productivité par $\mathrm{m}^{2}$ de surface déliégée au cours d'une rotation. Par ailleurs, la surface déliégée (sdl) est très fortement corrélée au DBH (Fig. 2c) selon la relation :

$$
\text { sdl }=0,0188 \mathrm{DBH}^{1,4083} \text { avec } r=0,991 * * *(\alpha=0,001)(14)
$$

témoignant que ces deux variables sont fortement liées.
En ce qui concerne les autres organes, les données des 12 arbres échantillons (Fig. 3) ont permis de mettre en évidence une forte corrélation entre leur biomasse (Y) et l'âge (A) de l'arbre (calculé à l'aide de la relation 10). Le tableau II regroupe les modèles d'ajustement retenus ainsi que leurs statistiques. Les équations de régression ajustées (relations 15 à 21) sont de type polynomial pour les feuilles, les rameaux et les racines, de type parabolique pour le bois et le liège des branches et de type puissance pour le bois et le liège mâle du tronc avec des coefficients de détermination $\left(R^{2}\right)$ très élevés.

Pour appréhender l'effet de l'âge de l'arbre sur les accroissements moyens en biomasse des différents organes durant la 

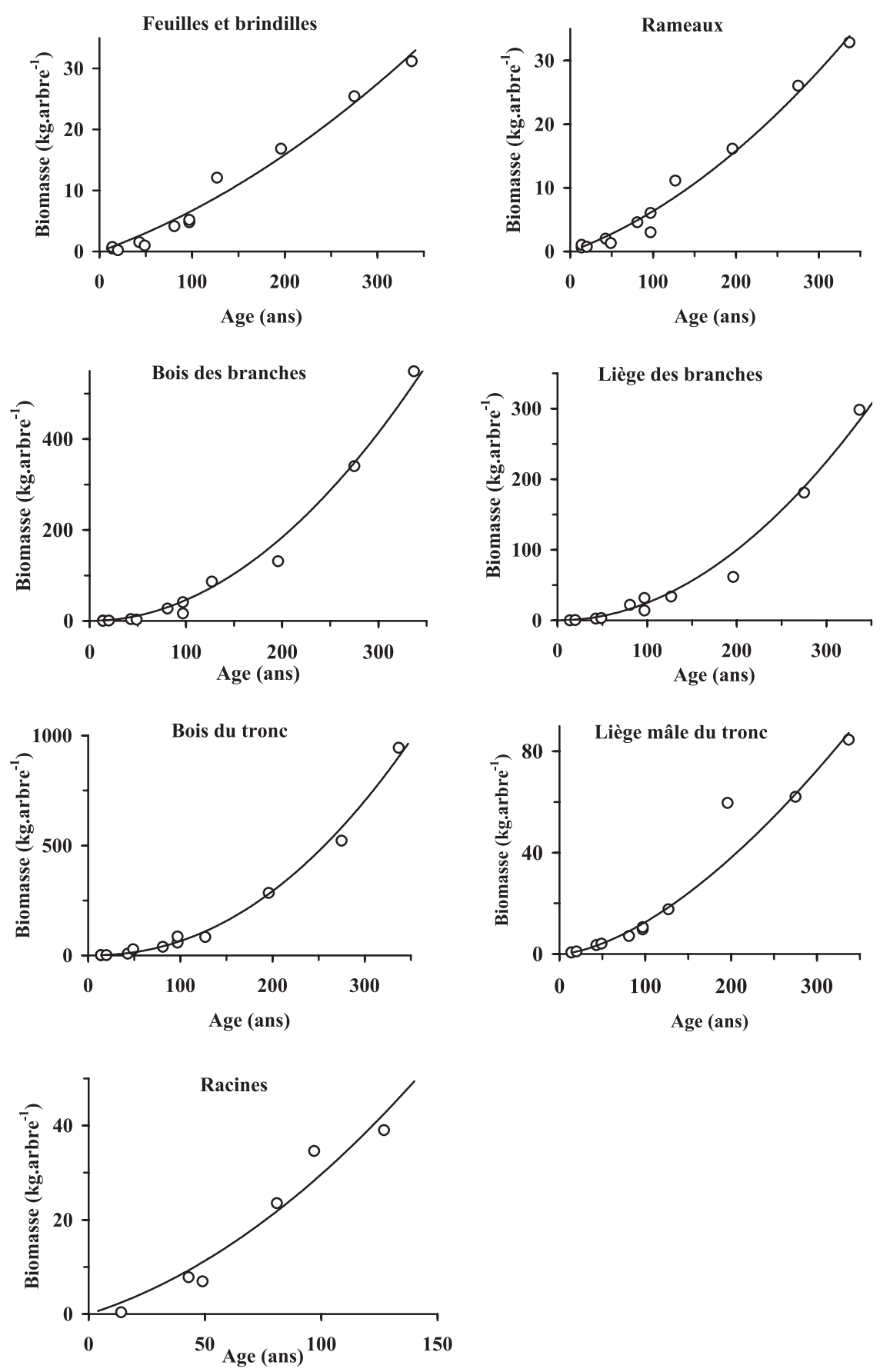

Figure 3. Relations entre les biomasses par compartiment et l'âge pour les 12 chênes lièges de Kroumirie (Tunisie) étudiés.

dernière rotation de 12 ans et leurs contributions relatives à l'accroissement en biomasse total, les tarifs intermédiaires (relations 5, 12 et 15 à 21) ont été appliqués à 3 arbres de 8,7, 20 et $60 \mathrm{~cm}$ de DBH respectifs. Les résultats consignés au tableau III montrent une forte augmentation de l'accroissement en biomasse total et une baisse du taux de croissance $R G R$ avec l'âge. À 53 ans, la biomasse de l'arbre s'accroît de $2,1 \mathrm{~kg} \mathrm{MS} \cdot \mathrm{an}^{-1}$ soit un $R G R$ de $2,14 \% \cdot \mathrm{an}^{-1}$. Cet accroissement passe à $4,7 \mathrm{~kg} \mathrm{MS} \cdot \mathrm{an}^{-1}$ correspondant à un taux de croissance de $0,94 \% \cdot a^{-1}$ à 112 ans et il atteint $14,6 \mathrm{~kg} \mathrm{MS} \cdot \mathrm{an}^{-1}$ ce qui correspond à un $R G R$ de $0,34 \% \cdot \mathrm{an}^{-1}$ à 303 ans. La contribution relative des organes à l'accroissement total traduit une modification du schéma de répartition des assimilats avec l'âge. En effet, les parts relatives du liège de reproduction et du bois du tronc se sont nettement accrues passant, respectivement, de 14 à $25,5 \%$ et de 28 à $34 \%$ aux dépens de tous les autres compartiments. 
Table III. Contributions relatives des différents compartiments à l'accroissement en biomasse total pour trois chênes lièges de Kroumirie (Tunisie) en fin de rotation et d'âges croissants : calculs à partir des biomasses estimées.

\begin{tabular}{|c|c|c|c|}
\hline Âge de l'arbre en fin de rotation (ans) & 53 & 112 & 303 \\
\hline DBH sous liège $(\mathrm{cm})$ & 8,7 & 20 & 60 \\
\hline Biomasse en début de rotation ( $\mathrm{kgMS}$ ) & 37,9 & 191,8 & 1547,9 \\
\hline Biomasse en fin de rotation (kgMS) & 63,0 & 248,3 & 1723,4 \\
\hline Accroissement en biomasse total $\left(\mathrm{kgMS} \cdot \mathrm{an}^{-1}\right)$ & 2,09 & 4,71 & 14,62 \\
\hline Taux de croissance $R G R\left(\% \cdot \mathrm{an}^{-1}\right)$ & 2,14 & 0,94 & 0,34 \\
\hline \multicolumn{4}{|c|}{ Contributions relatives à l'accroissement total en biomasse (\%) } \\
\hline Tronc & 48 & $\mathbf{5 4 , 5}$ & 62 \\
\hline Bois & 28 & 32 & 34 \\
\hline Liège de reproduction & 14 & 18 & 25,5 \\
\hline Liège mâle & 6 & 4,5 & 2,5 \\
\hline Houppier & 38 & 35,5 & 31 \\
\hline \multicolumn{4}{|l|}{ Branches } \\
\hline Bois & 21 & 21 & 19 \\
\hline Liège & 11 & 11 & 10 \\
\hline Rameaux & 3 & 2 & 1 \\
\hline Feuilles & 3 & 1,5 & 1 \\
\hline Racines & 14 & 10 & 7 \\
\hline
\end{tabular}

\subsection{Au niveau de la parcelle}

L'évolution des composants de la productivité primaire nette $\left(P N_{l}\right)$ le long de la séquence étudiée (Tab. IV) montre une forte baisse avec le taux de recouvrement des chênes lièges. En effet, la productivité primaire nette est de 4,0 à 5,2 $\mathrm{t} \mathrm{MS} \cdot \mathrm{ha}^{-1} \cdot \mathrm{an}^{-1}$ $\left(1791 \mathrm{à} 2341 \mathrm{kcal} \cdot \mathrm{m}^{-2} \cdot \mathrm{an}^{-1}\right)$ dans les parcelles en bon état (stade 1$)$ contre 0,7 à $1,7 \mathrm{t} \mathrm{MS} \cdot \mathrm{ha}^{-1} \cdot \mathrm{an}^{-1}\left(325 \mathrm{à} 760 \mathrm{kcal} \cdot \mathrm{m}^{-2} \cdot \mathrm{an}^{-1}\right)$ dans les autres parcelles. Les accroissements en biomasse montrent une baisse continue le long de la séquence de dégradation, passant de 1376 à $51 \mathrm{~kg} \mathrm{MS} \cdot \mathrm{ha}^{-1} \cdot \mathrm{an}^{-1}$ pour les troncs, de 892 à $70 \mathrm{~kg} \mathrm{MS} \cdot \mathrm{ha}^{-1} \cdot \mathrm{an}^{-1}$ pour les houppiers et de 263 à $44 \mathrm{~kg}$ $\mathrm{MS} \cdot \mathrm{ha}^{-1} \cdot \mathrm{an}^{-1}$ pour les racines. L'accroissement du liège de reproduction est passé de $456 \mathrm{~kg} \mathrm{MS} \cdot \mathrm{ha}^{-1} \cdot \mathrm{an}^{-1}$ dans la parcelle « $\mathrm{CR} 1$ » à $5 \mathrm{~kg} \mathrm{MS} \cdot \mathrm{ha}^{-1} \cdot \mathrm{an}^{-1}$ dans la parcelle « CR2 ». Les chutes totales de litière ont été de 2,26 à 2,85 t MS $\cdot \mathrm{ha}^{-1} \cdot \mathrm{an}^{-1}$ dans les parcelles du stade 1 contre 0,56 à $0,91 \mathrm{t} \mathrm{MS} \cdot \mathrm{ha}^{-1} \cdot \mathrm{an}^{-1}$ dans les parcelles dégradées. Les feuilles représentent 78 et $76 \%$ des chutes totales de litière dans les parcelles «BM1» et « AD1». Dans la station du Col des Ruines (CR), les proportions sont de $59 \%$ dans « CR1 » et de $90 \%$ dans « CR2 ». La production annuelle d'inflorescences et de glands a chuté de manière continue et hautement significative le long de la séquence, passant de 579 à $6 \mathrm{~kg} \mathrm{MS} \cdot \mathrm{ha}^{-1} \cdot \mathrm{an}^{-1}$ dans « CR1 » et « CR2 » respectivement. La production moyenne d'inflorescences et de glands par arbre (bra) (obtenue en divisant la production de la parcelle par le nombre d'arbres) est passée de 1,1 à $0,03 \mathrm{~kg} \mathrm{MS} \cdot \mathrm{an}^{-1}$ dans « $\mathrm{CR} 1$ » et « $\mathrm{CR} 2$ » respectivement. Elle augmente de manière significative avec l'âge $(\mathrm{A})$ du peuplement selon une loi exponentielle (Fig. 4) :

$$
\text { bra }=16\left(\mathrm{e}^{0,038 \mathrm{~A}}-1\right) \text { avec } r=0,886^{*}(\alpha=0,05)
$$

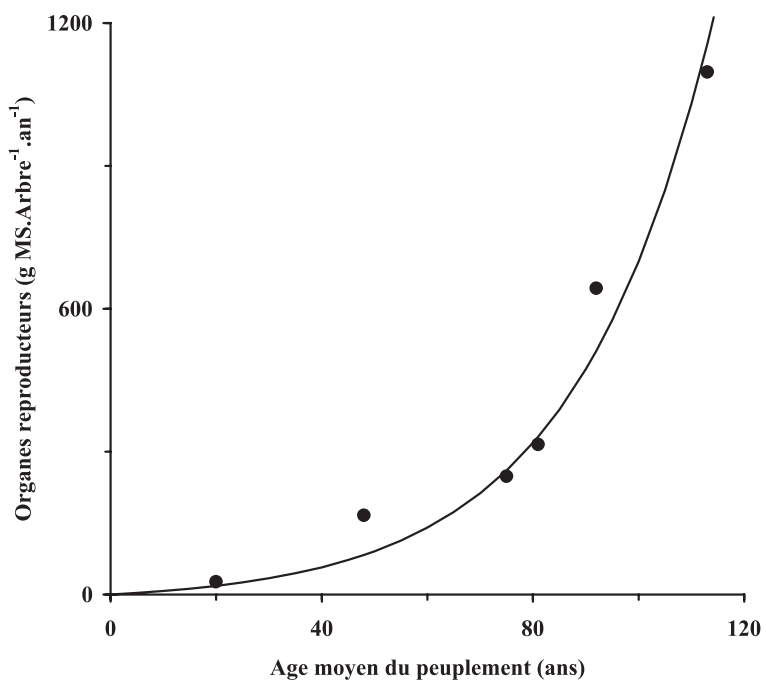

Figure 4. Relation entre la biomasse produite par les organes reproducteurs par arbre et l'âge moyen des peuplements dans les six parcelles d'étude représentant différents stades de dégradation de la suberaie à cytise de Kroumirie (Tunisie).

Le tableau $\mathrm{V}$ regroupe les biomasses pérennes des différentes parcelles en début et en fin de rotation et la figure 5 illustre l'évolution de leur taux de croissance $R G R$ avec l'âge du peuplement. Le liège du tronc a le plus fort $R G R$ (compris entre 8 et $5,8 \% \cdot \mathrm{an}^{-1}$ ) suivi du bois du tronc et des racines ayant des $R G R$ très similaires variant de 6,8 à $1,8 \% \cdot \mathrm{an}^{-1}$ puis celui des houppiers dont le $R G R$ ne dépasse pas $2,8 \% \cdot \mathrm{an}^{-1}$. L'augmentation de l'âge entraîne une baisse continue et modérée du taux de croissance pour le 
Table IV. Caractéristiques de peuplement et composants de la productivité primaire nette du chêne liège dans les six parcelles d'étude représentant différents stades de dégradation de la suberaie à cytise de Kroumirie (Tunisie).

\begin{tabular}{|c|c|c|c|c|c|c|}
\hline Parcelle et stade de dégradation & CR1 & BM1 & $\mathrm{AD} 1$ & $\mathrm{AD} 2$ & $\mathrm{BM} 2$ & $\mathrm{CR} 2$ \\
\hline Nombre d'arbres $\cdot \mathrm{ha}^{-1}$ & 528 & 723 & 322 & 258 & 301 & 223 \\
\hline Âge moyen (ans) & 113 & 81 & 92 & 75 & 48 & 20 \\
\hline Recouvrement des chênes lièges (\%) & 71,1 & 55,1 & 31,8 & 18,3 & 9,3 & 1,7 \\
\hline Surface terrière $\left(\mathrm{m}^{2} \cdot \mathrm{ha}^{-1}\right)$ & 32,9 & 26,4 & 14,8 & 8,51 & 4,97 & 0,99 \\
\hline$P N_{1}\left(\mathrm{t} \mathrm{MS} \cdot \mathrm{ha}^{-1} \cdot \mathrm{an}^{-1}\right)$ & 4,77 & 5,20 & 3,98 & 1,69 & 1,39 & 0,72 \\
\hline$B\left(\mathrm{kcal} \cdot \mathrm{m}^{-2} \cdot \mathrm{an}^{-1}\right)$ & 1129 & 1059 & 550 & 350 & 245 & 74 \\
\hline Litières $\left(\mathrm{kcal} \cdot \mathrm{m}^{-2} \cdot \mathrm{an}^{-1}\right)$ & 1017 & 1282 & 1241 & 410 & 379 & 251 \\
\hline
\end{tabular}

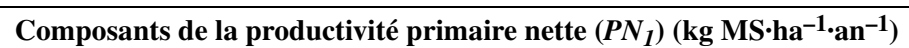

Accroissement en biomasse $(\Delta B)$

Troncs

Bois

Liège de reproduction

Liège mâle
811

456

109
738

361

120

389
206
58

\section{2}

157

55

35

40

Houppiers

Branches

\section{Bois \\ Liège}

Rameaux

Feuilles+brindilles

Racines

Total

Litières

Feuilles

Bois mort

Organes reproducteurs

Total

517

287

48

40

240

2508

1336

345

579

2260

\section{6}

269

56

50

263

2353

2222

399

228

2849

254
138
26
23
128
1222

1222

2095

455

207

2757

\section{3}

88

19

18

90

778

674

674
173

64

911

$\begin{array}{cc}115 & 29 \\ 63 & 16 \\ 19 & 12 \\ 19 & 13 \\ 82 & 44 \\ \mathbf{5 4 5} & \mathbf{1 6 5}\end{array}$

$616 \quad 501$

$177 \quad 50$

$50 \quad 6$

$843 \quad 557$

CR : col des Ruines ; BM : Ben Métir ; AD : Ain Debba.

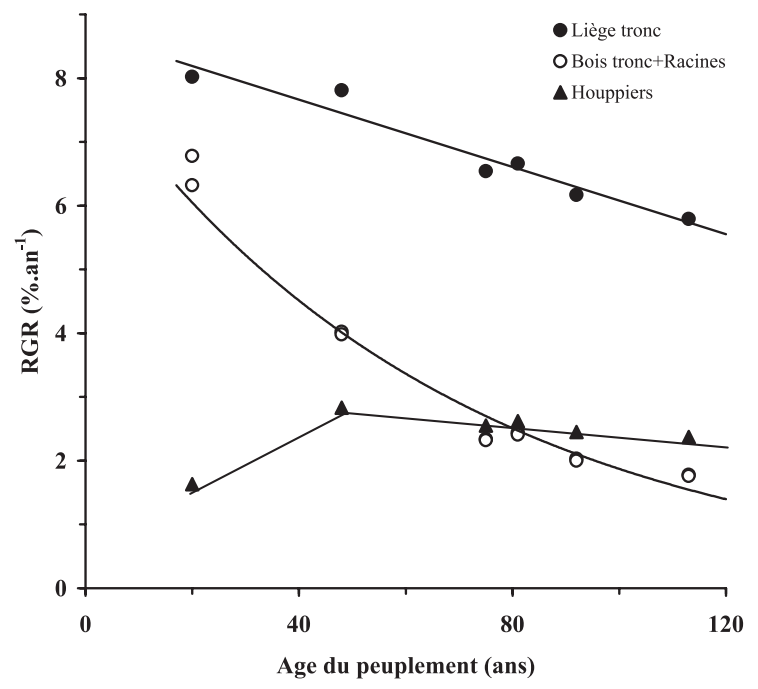

Figure 5. Relations entre les taux de croissance relatifs par compartiment $(R G R)$ et l'âge moyen des peuplements dans les six parcelles d'étude représentant différents stades de dégradation de la suberaie à cytise de Kroumirie (Tunisie). liège du tronc, une forte chute jusqu'à 75 ans pour le bois du tronc et les racines et un accroissement pour les houppiers chez les très jeunes peuplements suivi d'une diminution très légère. Les équations de régression suivantes ont été établies :

- liège du tronc :

$\mathrm{Y}=-0,0264 \mathrm{~A}+8,716$ avec $r=-0,972 * *(\alpha=0,01)$

- bois du tronc et racines :

$\mathrm{Y}=8,1102 \mathrm{e}^{-0,0147 \mathrm{~A}}$ avec $r=-0,945^{* * *}(\alpha=0,001)$

- houppiers :

$\mathrm{Y}=-0,0071 \mathrm{~A}+3,147$ avec $r=-0,961 * *(\alpha=0,01)(25)$ l'équation (25) ne s'applique que pour les peuplements d'âge supérieur à 40 ans.

Pour chercher des indicateurs de la dégradation, nous avons considéré comme facteurs de la productivité primaire deux variables caractéristiques de la structure des peuplements : le taux de recouvrement des chênes lièges (TR) et leur surface terrière $(\mathrm{G})$. La figure $6 \mathrm{a}$ montre une relation linéaire entre ces deux variables d'équation :

$$
\mathrm{TR}=2,1278 \mathrm{G} \text { avec } r=0,999 * * *(\alpha=0,001)
$$


Table V. Biomasses des organes pérennes en fin $\left(\mathrm{B}_{2}\right.$ au temps $\left.\mathrm{t}_{2}\right)$ et en début $\left(\mathrm{B}_{1}\right.$ au temps $\left.t_{1} ; t_{1}=t_{2}-12\right)$ de rotation dans les six parcelles d'étude représentant différents stades de dégradation de la suberaie à cytise de Kroumirie (Tunisie).

\begin{tabular}{|c|c|c|c|c|c|c|c|c|c|c|c|c|}
\hline \multirow[t]{3}{*}{ Parcelles } & \multicolumn{2}{|c|}{ CR1 } & \multicolumn{2}{|c|}{$\mathrm{AD} 1$} & \multicolumn{2}{|c|}{ BM1 } & \multicolumn{2}{|c|}{$\mathrm{AD} 2$} & \multicolumn{2}{|c|}{ BM2 } & \multicolumn{2}{|c|}{$\mathrm{CR} 2$} \\
\hline & \multicolumn{12}{|c|}{ Biomasses $\left(t \cdot h^{-1}\right)$} \\
\hline & $\mathrm{B}_{2}$ & $\mathrm{~B}_{1}$ & $\mathrm{~B}_{2}$ & $\mathrm{~B}_{1}$ & $\mathrm{~B}_{2}$ & $\mathrm{~B}_{1}$ & $\mathrm{~B}_{2}$ & $\mathrm{~B}_{1}$ & $\mathrm{~B}_{2}$ & $\mathrm{~B}_{1}$ & $\mathrm{~B}_{2}$ & $\mathrm{~B}_{1}$ \\
\hline \multicolumn{13}{|l|}{ Troncs } \\
\hline Bois & 50,6 & 40,9 & 21,6 & 17,0 & 34,9 & 26,1 & 11,8 & 8,94 & 4,92 & 3,04 & 0,77 & 0,36 \\
\hline Liège & 13,5 & 6,76 & 6,06 & 2,89 & 10,5 & 4,72 & 3,49 & 1,59 & 1,78 & 0,70 & 0,33 & 0,13 \\
\hline Houppiers & 48,8 & 36,7 & 21,2 & 15,8 & 34,6 & 25,3 & 11,9 & 8,79 & 5,91 & 4,21 & 2,43 & 1,99 \\
\hline Racines & 25,8 & 20,9 & 11,0 & 8,67 & 17,8 & 13,3 & 6,03 & 4,56 & 2,50 & 1,55 & 0,39 & 0,17 \\
\hline
\end{tabular}

CR : col des Ruines ; BM : Ben Métir ; AD : Ain Debba ; $\mathrm{B}_{2}$ : biomasse en fin de rotation (temps $t_{2}$ ); $\mathrm{B}_{1}$ : biomasse en début de rotation (temps $t_{1}=t_{2}-12$ ).

Table VI. Équations de régression établies entre la productivité primaire nette $\left(\mathrm{PN}_{1}\right.$ en $\left.\mathrm{kg} \mathrm{MS} \cdot \mathrm{ha}^{-1} \cdot \mathrm{an}^{-1}\right)$ ainsi que celle de ses composants et la surface terrière $\left(\mathrm{G} \mathrm{en} \mathrm{m}^{2} \cdot \mathrm{ha}^{-1}\right)$ dans les six parcelles d'étude $(n=6)$ représentant les différents stades de dégradation de la suberaie à cytise de Kroumirie (Tunisie).

\begin{tabular}{|c|c|c|c|c|c|}
\hline $\begin{array}{l}\text { Productivité primaire } \\
\text { nette }\left(\mathrm{PN}_{1}\right) \text { et ses composants }\end{array}$ & Modèle de régression & $R^{2}$ & $s$ & $r$ & Relations \\
\hline$P N_{l}$ & $Y=-5,237 X^{2}+323,769 X$ & 0,987 & 484,1 & 0,975 & (26) \\
\hline \multicolumn{6}{|l|}{ Biomasse vivante } \\
\hline Bois du tronc & $Y=26,109 X$ & 0,996 & 34,2 & 0,996 & (27) \\
\hline Liège du tronc & $Y=17,631 X$ & 0,999 & 10,4 & 0,999 & (28) \\
\hline Bois des branches & $\mathrm{Y}=17,082 \mathrm{X}$ & 0,991 & 32,9 & 0,991 & (29) \\
\hline Liège des branches & $Y=9,369 X$ & 0,993 & 16,1 & 0,993 & (30) \\
\hline Rameaux & $Y=1,768 X$ & 0,929 & 9,11 & 0,943 & $(31)$ \\
\hline Feuilles & $Y=1,542 X$ & 0,911 & 9,85 & 0,911 & $(32)$ \\
\hline Racines & $Y=8,553 X$ & 0,961 & 35,3 & 0,966 & (33) \\
\hline Total & $Y=82,053 X$ & 0,993 & 143,9 & 0,993 & (34) \\
\hline \multicolumn{6}{|l|}{ Litières } \\
\hline Feuilles & $Y=-4,0601 X^{2}+180,53 X$ & 0,944 & 413,6 & 0,877 & (35) \\
\hline Bois mort & $Y=-0,8394 X^{2}+38,026 X$ & 0,974 & 60,1 & 0,940 & (36) \\
\hline Organes reproducteurs & $Y=0,448 X^{2}$ & 0,937 & 74,3 & 0,944 & (37) \\
\hline Total & $Y=-4,559 X^{2}+222,91 X$ & 0,964 & 451,6 & 0,922 & (38) \\
\hline
\end{tabular}

$\mathrm{X}$ : surface terrière de la parcelle $\left(\mathrm{m}^{2} \cdot \mathrm{ha}^{-1}\right) ; \mathrm{Y}:$ accroissement en biomasse $\left(\mathrm{kg} \mathrm{MS} \cdot \mathrm{ha}^{-1} \cdot \mathrm{an}^{-1}\right) ; R^{2}:$ coefficient de détermination ; $s$ écart type résiduel $; r^{\prime}$ : coefficient de corrélation entre $\mathrm{Y}$ estimée et $\mathrm{Y}$ prédite.

Les dépendances entre la surface terrière et les composants de la productivité primaire des parcelles étudiées (Figs. 6b, 6c, 6d et Tab. VI) sont linéaires pour les accroissements de la biomasse vivante et curvilinéaires pour la litière. Les coefficients de détermination des relations établies sont très élevés pour les différents organes. Les résultats montrent aussi que la dégradation a affecté davantage l'accroissement de la biomasse vivante que celui de la matière organique morte. En effet, on a observé une chute continue de l'accroissement de la biomasse vivante avec la baisse de la surface terrière, à raison de $82 \mathrm{~kg} \mathrm{MS} \cdot \mathrm{ha}^{-1} \cdot \mathrm{an}^{-1}$, alors que la litière n'a accusé une forte baisse qu'au-dessous de $15 \mathrm{~m}^{2} \cdot \mathrm{ha}^{-1}$ de surface terrière (Fig. 6b).

La répartition de l'accroissement en biomasse des différents organes non assimilateurs (en \%) pour les six parcelles est présentée à la figure 7. Pour l'ensemble des parcelles, la contribution a été, en moyenne respectivement de 35,54 et $11 \%$ pour les troncs, les houppiers et les racines. Seuls le liège mâle, le bois et le liège des branches n'ont pas été très touchés par la dégradation ; tous les autres compartiments l'ont été plus ou moins, en particulier dans les parcelles «BM2 » et «CR2». En effet, on a enregistré, dans ces parcelles, une baisse sensible de la production de liège de reproduction, de l'accroissement du bois de tronc et de la biomasse des organes reproducteurs. À l'opposé, l'accroissement en biomasse des rameaux et des racines a augmenté nettement, passant de 12 et $8 \%$ respectivement dans la parcelle la mieux conservée « $\mathrm{CR} 1$ » à 30 et $21 \%$ dans la parcelle la plus dégradée « $\mathrm{CR} 2 »$.

\section{DISCUSSION}

L'évaluation de la productivité primaire des chênes lièges dans le présent travail est basée sur 12 arbres échantillons de 2 

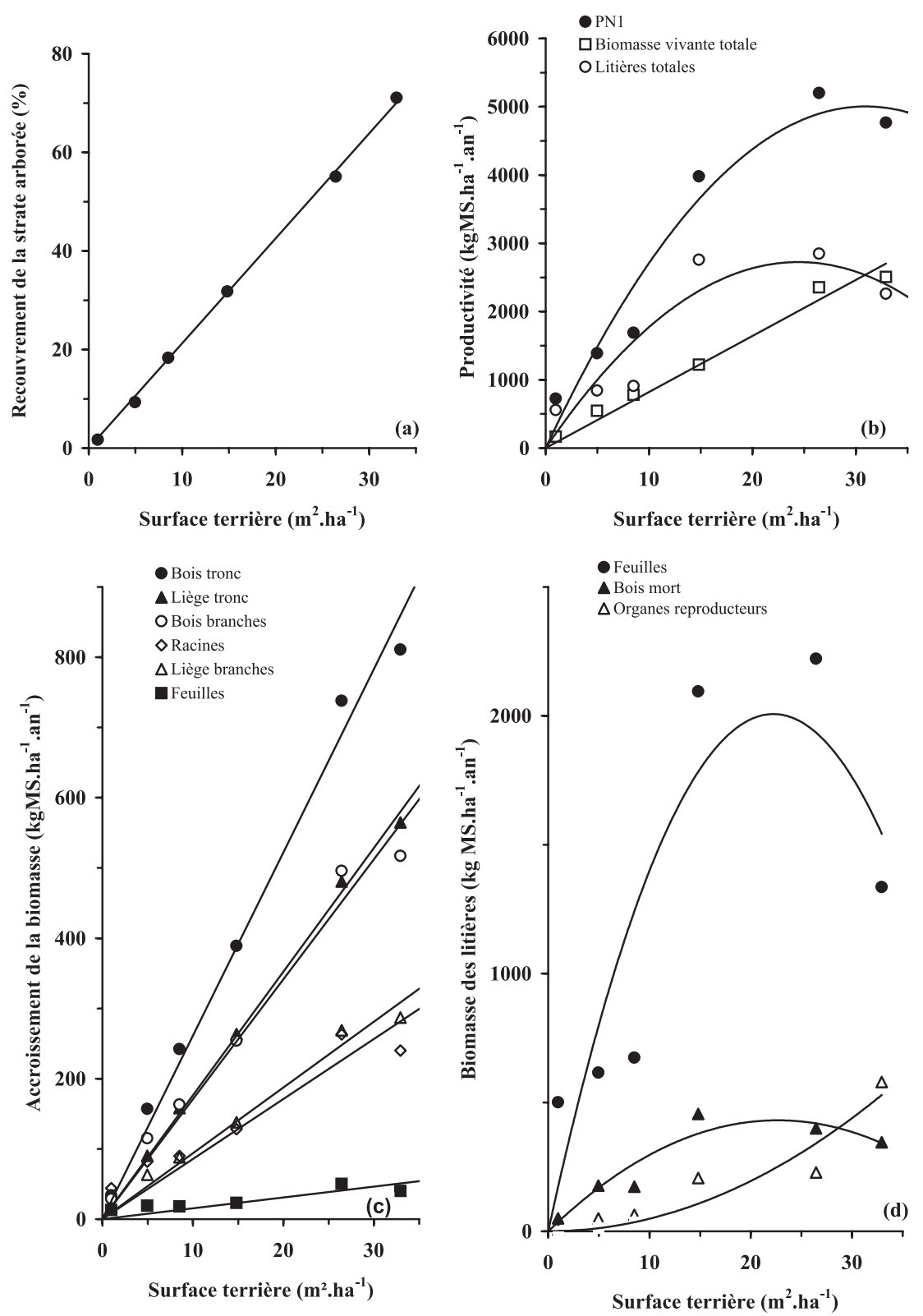

Figure 6. Relations avec la surface terrière (a) du recouvrement de la strate arborée, (b) des composants de la productivité primaire nette $\left(\mathrm{PN}_{1}\right)$, (c) des accroissements en biomasse des compartiments pérennes, (d) des composants de la litière. Les observations concernent les six parcelles d'étude représentant différents stades de dégradation de la suberaie à cytise de Kroumirie (Tunisie).

à $67,5 \mathrm{~cm}$ de DBH sous liège (Tab. I). Une telle gamme de diamètres couvre à la fois celle des parcelles étudiées $(2$ à $52 \mathrm{~cm})$ avec des DBH moyens de 3 à $20 \mathrm{~cm}$ selon les parcelles [61] ainsi que celle de l'ensemble de la suberaie tunisienne allant jusqu'à $68 \mathrm{~cm}$ avec un DBH moyen de $14 \mathrm{~cm}$ [11]. Cet échantillon semble de taille acceptable comparé au nombre d'arbres échantillons d'études antérieures qui varie de 5 [38] à 13 [39].

L'accroissement du bois de chêne liège, basé sur le nombre et la largeur des cernes annuels, a été étudié sur des sections transversales de tronc (rondelles) prélevées à $1.30 \mathrm{~m} \mathrm{[38]} \mathrm{ou}$ bien à la fois sur des rondelles de tronc et des carottes prélevées à la tarrière de Pressler à la même hauteur [2, 7]. Dans le présent travail, nous avons opté pour la technique des rondelles en raison de l'inclinaison des troncs (pente des stations de 20 à $30 \%$ ) responsable de la formation de bois de tension à la face supé- rieure et d'une excentricité de la section [18, 31, 50]. Certains auteurs prennent pour âge de l'arbre le nombre des cernes à 1,30 m [29, 38]. Nos résultats (Fig. 1) montrent que l'âge réel des arbres est mieux reflété par le nombre des cernes à la base. La relation (10), de type puissance, entre l'âge et le DBH est de même forme et très proche de celle reliant l'âge à la circonférence du tronc à $1,30 \mathrm{~m}[29,38]$. La figure 1 semble indiquer qu'aux forts diamètres, l'âge des arbres est surestimé par la relation (10). L'analyse de l'ajustement réalisé donne un coefficient de corrélation de 0,992 entre l'âge déterminé et l'âge prédit, un écart type résiduel de 0,046 ans et un coefficient de variation de $0,08 \%$, témoignant de la validité de cette relation, du moins pour les arbres de diamètre inférieur à $20 \mathrm{~cm}$. La relation (11) montre que l'accroissement radial annuel moyen du bois à 1,30 m est proche de $1 \mathrm{~mm}$ dans l'intervalle de DBH de 


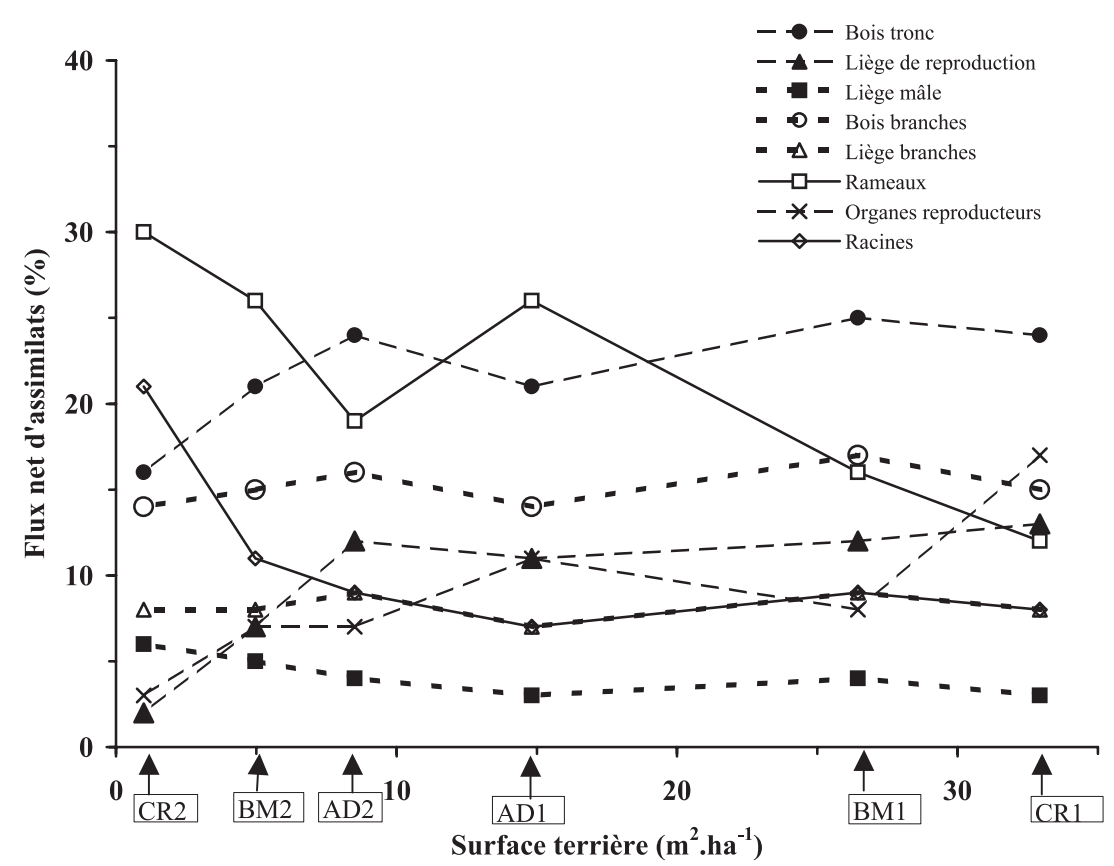

Figure 7. Allocation des assimilats aux différents organes non assimilateurs en fonction de la surface terrière dans les six parcelles d'étude représentant les différents stades de dégradation de la suberaie à cytise de Kroumirie (Tunisie). (CR : col des Ruines; BM : Ben Métir; AD : Ain Debba).
0 à 20 cm (c'est-à-dire jusqu'à 112 ans). La largeur des cernes ligneux, mesurée sur 44 individus de la suberaie de Girona (Espagne) varie entre 1,2 et 3,7 mm.an ${ }^{-1}$ selon l'âge des arbres et la pluviosité de la station, avec une moyenne de $2 \mathrm{~mm} \cdot \mathrm{an}^{-1}$ [7], valeur comparable à celles des chênes lièges de la région de Sughero (Italie) [8]. La faible croissance radiale enregistrée pour les dix arbres abattus à proximité de la station $\mathrm{AD}$ pourrait être attribuée à la mauvaise répartition saisonnière des précipitations en Kroumirie ou à la faible réserve d'eau utile du sol et/ ou à la fertilité médiocre de cette station, en particulier sa faible teneur en azote [61]. L'accroissement moyen du liège dans nos parcelles est de $2,7 \mathrm{~mm} \cdot \mathrm{an}^{-1}$ au cours d'une rotation de 12 ans [61] qui, ajouté à celui du bois, porte la croissance radiale du tronc à $3,7 \mathrm{~mm} \cdot \mathrm{an}^{-1}$. Cet accroissement du liège est comparable à celui de la suberaie d'Ait Hatem au Maroc égal à 2,15 mm·an1 pour une même période de rotation [20] et semble compatible avec cette durée de rotation. En effet, l'accroissement du liège est de 3,3 à $4,1 \mathrm{~mm} \cdot \mathrm{an}^{-1}$ dans la suberaie portugaise pour une rotation de 8 à 9 ans $[22,23,51]$ et passe à $2,1 \mathrm{~mm} \cdot \mathrm{an}^{-1}$ pour une rotation de 14 à 18 ans dans la suberaie catalane d'Espagne [25] soulignant le ralentissement de la croissance du liège de reproduction avec le temps. En effet, l'accroissement du liège pendant les trois premières années qui suivent le déliégeage représente $48 \%$ de l'épaisseur atteinte en 8 ans [23] ; il atteint, au bout de 6 ans, $75 \%$ de l'épaisseur qu'il aura à 9 ans pour les classes de haute fertilité et $66 \%$ de celle atteinte en 12 ans dans les stations défavorables [62].

Nos résultats (Fig. 2) attestent que le DBH et la surface déliégée sont deux variables liées et très hautement explicatives de la production de liège par individu. Des résultats comparables ont été obtenus dans les suberaies du Portugal [22]. De même, la production de liège calculée à l'aide de la relation (12) est comparable à celle obtenue pour ces suberaies portugaises, soit 0,6 à $6,4 \mathrm{~kg} \cdot \operatorname{arbre}^{-1} \cdot \mathrm{an}^{-1}$ pour un $\mathrm{DBH}$ sous liège variant entre 16,3 et $80,7 \mathrm{~cm}$ [22]. D'autre part, la productivité moyenne du liège sec est de $7,9 \mathrm{~kg} \cdot \mathrm{m}^{-2}$ de surface déliégée au cours d'une rotation de 12 ans (relation 13). Cette productivité dépend de la densité du liège, liée elle-même à la fertilité de la station [62] et à la durée de rotation [23]. Des productivités moyennes de $8,8 \mathrm{~kg} \cdot \mathrm{m}^{-2}$ de surface déliégée ont été enregistrées au Portugal $[23,48]$ et en Espagne [25, 26] pour des rotations variant entre 8 et 18 ans.

Les très fortes corrélations entre la biomasse des compartiments non exploités et l'âge des arbres échantillons (Fig. 3) et la qualité des ajustements obtenus (Tab. II) sont en faveur de l'hypothèse avancée plus haut, à savoir que ces arbres échantillons forment une chrono-séquence et représentent ainsi différents stades de développement d'un même arbre au cours du temps. Ces résultats attestent que l'âge de l'arbre est une variable très hautement explicative de la biomasse des chênes lièges au niveau de l'individu au même titre que le DBH [61]. La forte liaison entre ces deux variables (Fig. 1) est en accord avec celle obtenue sur une suberaie de Sicile [38]. La plupart des tarifs intermédiaires de biomasse des essences forestières utilisent le DBH comme variable prédictive [3, 24, 27, 34-36, 38-40, 58, 63]. Des tarifs combinant la circonférence à $1,30 \mathrm{~m}$ et l'âge ont aussi été établis [52].

L'accroissement en biomasse à 53 ans (Tab. III) est inférieur à ceux enregistrés chez de jeunes chênes lièges et chênes verts (Q. ilex) de Sicile agés de 31 ans $[35,37]$ alors que celui atteint à 112 ans est comparable à celui de l'arbre moyen d'un taillis de chênes verts de 150 ans du sud de la France [32]. Ces différences pourraient être attribuées à l'espèce mais aussi à la fertilité de la station. La baisse du taux de croissance de la biomasse avec l'âge de l'arbre (Tab. III) a été également mise en évidence chez le chêne vert $[32,35]$.

Les contributions relatives des différents organes à l'accroissement en biomasse total (Tab. III), soulignent une modification de la taille des différents « réservoirs » d'assimilats avec l'âge de l'arbre. Il apparaît notamment que l'exploitation régulière du 
Table VII. Comparaison des accroissements en biomasse et des chutes de litières pour différents peuplements de chênes du bassin occidental de la Méditerranée (d'après différents auteurs).

\begin{tabular}{|c|c|c|c|c|c|c|c|}
\hline \multirow{2}{*}{ Espèces } & \multirow{2}{*}{ Pays (région) } & \multirow{2}{*}{$\begin{array}{c}\text { Densité } \\
\left(\text { arbres }^{\prime} \text { ha }^{-1}\right)\end{array}$} & \multirow{2}{*}{ Âge (ans) } & \multicolumn{3}{|c|}{ Accroissement de la biomasse aérienne $\left(\mathrm{t} \cdot \mathrm{ha}^{-1} \cdot \mathrm{an}^{-1}\right)$} & \multirow{2}{*}{ Références } \\
\hline & & & & Organes pérennes & Litière & Total & \\
\hline \multicolumn{8}{|c|}{ Quercus ilex } \\
\hline & Italie (Sicile) & 968 & 31 & 4,58 & 3,13 & 7,71 & [37] \\
\hline & France (Sud) & 1441 & 150 & 3,10 & 3,90 & 7,00 & [41] \\
\hline & $" \quad "$ & 4171 & 42 & 1,94 & 3,76 & 5,70 & [55] \\
\hline \multicolumn{8}{|c|}{ Quercus suber } \\
\hline & $\begin{array}{c}\text { Italie (Sicile) } \\
\text { Tunisie }\end{array}$ & 345 & 31 & 1,10 & 3,60 & 4,70 & {$[38]$} \\
\hline & (Kroumirie) & 723 & 81 & 2,10 & 2,85 & 4,95 & Présente étude \\
\hline & BM1 & 528 & 113 & 2,37 & 2,26 & 4,63 &,$\quad$, \\
\hline & CR1 & 322 & 92 & 1,10 & 2,76 & 3,86 & $"$ \\
\hline & $\mathrm{AD} 1$ & & & & & & \\
\hline \multicolumn{8}{|c|}{ Quercus coccifera } \\
\hline & France (Sud) & faible & 17 & 1,40 & 2,30 & 3,70 & [54] \\
\hline & $" \quad "$ & dense & 30 & 1,10 & 2,60 & 3,70 & $"$ \\
\hline
\end{tabular}

CR : col des Ruines ; BM : Ben Métir ; AD : Ain Debba.

liège active de façon continue ce « réservoir » contrairement au liège mâle, résultat conforme à celui obtenu sur les suberaies portugaises [48]. On constate enfin que l'accroissement en biomasse du bois du tronc est nettement accru entre 53 et 303 ans aux dépens des autres organes.

Les résultats au niveau de la parcelle (Tab. IV) montrent que pour les suberaies les moins dégradées, la productivité totale $\left(P N_{l}\right)$ des chênes lièges est de 4,0 à 5,2 $\mathrm{t} \mathrm{MS} \cdot \mathrm{ha}^{-1} \cdot \mathrm{an}^{-1}$ équivalent à 1791 à $2341 \mathrm{kcal} \cdot \mathrm{m}^{-2} \cdot \mathrm{an}^{-1}$. L'accroissement en biomasse des organes souterrains est de 0,13 à $0,26 \mathrm{t} \cdot \mathrm{ha}^{-1} \cdot \mathrm{an}^{-1}$ dans ces parcelles. Les données relatives à ce compartiment dans les chênaies méditerranéennes ne sont pas disponibles dans la littérature. Néanmoins, ces valeurs sont nettement inférieures à celles de peuplements de $Q$. petraea de Belgique $\left(1,68 \mathrm{t} \cdot \mathrm{ha}^{-1} \cdot \mathrm{an}^{-1}\right)$ [13] et de Fagus sylvatica $\left(1,6\right.$ à 1,86 th.ha $\left.{ }^{-1} \cdot \mathrm{an}^{-1}\right)$ de Belgique [15] et de France [34]. Les valeurs de la présente étude sont au-dessous de la réalité car il n'a pas été tenu compte de l'accroissement en biomasse de la souche ni du turn-over des racines fines qui s'élèverait à 0,5 à $0,6 \mathrm{t} \cdot \mathrm{ha}^{-1} \cdot \mathrm{an}^{-1}$ pour un peuplement de hêtres de 100 à 116 ans [34].

Le tableau VII montre une comparaison entre la productivité aérienne des forêts de chênes lièges et de chênes verts et des garrigues à chênes kermès $(Q$. coccifera) du bassin méditerranéen. On remarque que la productivité aérienne du chêne liège est intermédiaire entre celle du chêne vert (Quercus ilex) et celle du chêne kermès (Quercus coccifera) aussi bien pour l'accroissement de la biomasse vivante que pour la chute de litière. L'accroissement de la biomasse aérienne des chênes lièges de Sicile est comparable à celui de la parcelle «AD1» de densité voisine, mais est inférieur à ceux des parcelles « $\mathrm{CR} 1$ » et « BM1 » de densité et d'âge nettement supérieurs. Les chutes totales de litière dans nos parcelles sont sensiblement inférieures à celles du peuplement de Sicile à cause du bois mort qui atteint 1,25 $\mathrm{t} \mathrm{MS} \cdot \mathrm{ha}^{-1} \cdot \mathrm{an}^{-1}$ dans ce dernier cas. La litière de feuilles des parcelles « BM1 » et « AD1 » (Tab. IV) est comparable à celle du peuplement de Sicile qui s'élève à $2 \mathrm{t} \mathrm{MS} \cdot \mathrm{ha}^{-1} \cdot \mathrm{an}^{-1}$ [38] et sa proportion dans la litière totale ( 78 et $76 \%$ ) est comparable à celle de deux peuplements de la suberaie espagnole de Girona
[56]. Par contre, la litière de feuilles de la parcelle « CR1 » qui représente seulement $59 \%$ de la litière totale est anormalement faible : ceci est sans doute imputable à une attaque par Lymantria dispar en 1991. La biomasse des organes reproducteurs de la litière dans les parcelles en bon état (Tab. IV) est proche de celle des chênes lièges de Sicile $\left(423 \mathrm{~kg} \cdot \mathrm{ha}^{-1} \cdot \mathrm{an}^{-1}\right)$ [38] mais est sensiblement inférieure à celle du chêne vert laquelle varie entre 620 et $750 \mathrm{~kg} \cdot \mathrm{ha}^{-1} \cdot \mathrm{an}^{-1}[16,37,41]$.

L'accroissement en biomasse total du liège du tronc dans les parcelles en bon état varie entre 264 et $565 \mathrm{~kg}$ MS.ha ${ }^{-1} \cdot \mathrm{an}^{-1}$ dont 75 à $81 \%$ de liège de reproduction (Tab. IV). La seule donnée de référence citée dans la littérature est de $217 \mathrm{~kg}$ $\mathrm{MS} \cdot \mathrm{ha}^{-1} \cdot \mathrm{an}^{-1}$ pour le peuplement de Sicile [38], valeur proche de celle de la parcelle «AD1 ». L'accroissement en biomasse du liège de reproduction semble refléter, à la fois, les caractéristiques dendrométriques du peuplement et les qualités de la station. En effet, dans nos parcelles en bon état, la productivité annuelle du liège de reproduction est de $13,8 \mathrm{~kg} \mathrm{MS} \cdot \mathrm{m}^{-2} \mathrm{de}$ surface terrière (Tab. IV) pour des précipitations annuelles comprises entre 1140 et $1572 \mathrm{~mm}$; les calculs donnent pour deux peuplements de la suberaie de Girona, 12,9 et $8,9 \mathrm{~kg} \mathrm{MS} \cdot \mathrm{m}^{-2}$, mais pour des pluviométries annuelles respectives de 858 et $659 \mathrm{~mm}[44,56]$.

Les résultats de la figure 5 comme ceux obtenus au niveau de l'individu (Tab. III) soulignent l'effet stimulateur de l'exploitation régulière du liège du tronc sur la croissance de ce compartiment [48]. Ils montrent aussi des taux de croissance du bois du tronc et des racines très similaires. La baisse du taux de croissance du bois du tronc avec l'âge du peuplement est concordante avec les résultats obtenus sur des peuplements de $Q$. ilex d'âges différents [28, 36, 37, 41, 55].

Une forte liaison linéaire a été enregistrée entre le taux de recouvrement (TR) des chênes lièges et leur surface terrière $(G)$ (Fig. 6a) traduisant une baisse simultanée de ces deux paramètres le long de la séquence étudiée. Pour savoir si une telle relation peut être étendue à d'autres régions de la suberaie tunisienne, nous avons utilisé les données de l'Inventaire Forestier National [11] relatives aux peuplements de chêne liège pur du gouvernorat 


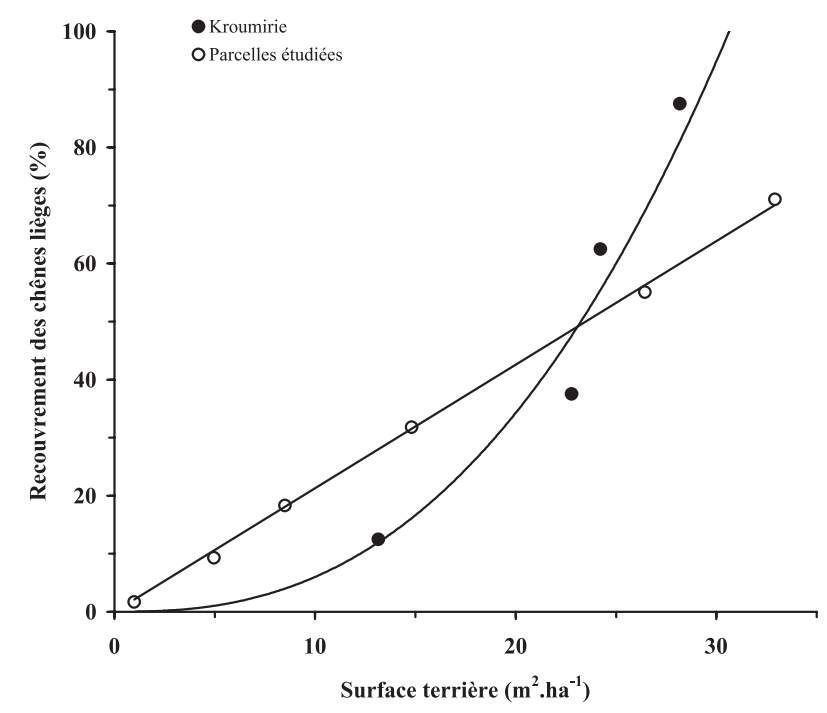

Figure 8. Relation entre le recouvrement et la surface terrière des peuplements de chêne liège pur du gouvernorat de Jendouba (Kroumirie, Tunisie) comparée à celle des parcelles étudiées.

de Jendouba couvrant 35600 ha. Nous avons calculé la surface terrière à partir des effectifs des arbres répartis en quatre classes de recouvrement de $25 \%$ chacune et en classes de DBH de $5 \mathrm{~cm}$. La figure 8 montre que ces deux paramètres dendrométriques peuvent être reliés par une fonction de type puissance d'équation :

$$
\mathrm{TR}=0,0186 \mathrm{G}^{2,5103} \text { avec } r=0,942
$$

différente de la forme linéaire de la relation (26). Ce résultat tend à prouver qu'une telle relation varie selon le peuplement, mais il faut noter que le taux de recouvrement n'a pas été estimé de la même façon dans les deux cas. L'extrapolation d'une telle relation à d'autres régions nécessite cependant quelques précautions.

En outre, les parcelles dégradées ont été caractérisées par une baisse du nombre d'arbres à l'hectare et de l'âge moyen du peuplement (Tab. IV). Ces modifications dendrométriques pourraient être une conséquence de coupes délictuelles portant davantage sur les arbres de forts diamètres comme nous l'avons déjà montré [61]. Par ailleurs, la figure 6 et le tableau VI témoignent que la surface terrière des chênes lièges et leur taux de recouvrement sont deux variables hautement explicatives des productivités primaires nettes totales et partielles de la strate arborée. Ces deux paramètres constituent ainsi, pour la région considérée, des critères quantitatifs de l'état du peuplement, résultats en accord avec ceux relatifs à la biomasse totale et compartimentale des chênes lièges [61]. La forme des relations atteste que l'accroissement de la biomasse vivante a été plus affecté que la production d'organes caducs par la diminution de la surface terrière.

L'accroissement en biomasse des organes non assimilateurs traduit le flux net d' assimilats exportés hors des feuilles et accumulés dans la biomasse. La figure 7 montre que le schéma de distribution des assimilats vers les organes pérennes a été affecté différemment selon les organes. En effet, au stade 2, les proportions d'assimilats orientés vers, le liège mâle le bois et le liège des branches sont du même ordre qu'au stade 1 alors qu'une baisse a été enregistrée dans le cas du liège de reproduction, du bois du tronc et des organes reproducteurs et qu'un accroissement sensible s'est manifesté pour les rameaux et les racines. De telles différences traduisent, vraisemblablement, des modifications dans la physiologie de l'arbre en rapport avec le rajeunissement des peuplements dégradés.

Remerciements : Le présent travail a été réalisé grâce à l'appui financier du Ministère de l'Enseignement Supérieur, de la Recherche et de la Technologie (UR 00/UR/09-02) et de l'IRESA (AR 140 6102) du Ministère de l'Agriculture, de l'Environnement et des Ressources Hydrauliques. Nous tenons à remercier la Direction Générale des Forêts et l'Arrondissement des Forêts de Ain Draham pour l'autorisation d'abattage des arbres échantillons et l'aide technique fournie.

\section{RÉFÉRENCES}

[1] Abid H., Selmi K., La suberaie tunisienne: importance et orientations pour une gestion durable, in: Actes sémin. médit. sur la régénération des forêts de chêne liège, Tabarka-Tunisie 22-24 octobre 1996, Ann. INRGREF Suppl., 1998, pp. 63-72.

[2] Aloui A., Recherches dendroclimatologiques en Kroumirie (Tunisie), Thèse Docteur Ingénieur, Univ. Aix-Marseille III, 1982.

[3] Bartelink H.H., Allometric relationships for biomass and leaf area of branch (Fagus sylvatica L.), Ann. Sci. For. 54 (1997) 159-168.

[4] Ben Jemaa M.H., Hasnaoui B., Le dépérissement du chêne liège (Quercus suber L.) en Tunisie, in: Coll. Nat. sur le dépérissement des Forêts au Maroc, CNRF Rabat-Maroc 28 et 29 février 1996.

[5] Benzyane M., La suberaie marocaine: produit économique et social à développer, in: Actes sémin. médit. sur la régénération des forêts de chêne liège, Tabarka-Tunisie, 22-24 octobre 1996, Ann. INRGREF Suppl., 1998, pp. 12-21.

[6] Bouchon J., Les tarifs de cubage, INRA et INGREF Nancy (France), 1974, 59 p. + annexes.

[7] Caritat A., Molinas M., Oliva M., El crecimiento radial del alcornoque en cinto parcellas de alconocal de Girona, Scientia gerundensis 18 (1992) 73-83.

[8] Cambini A., Effetti della defogliazione anticipata sull acrescimento della quercia da sughero (Quecus suber L.), Stazionesperimentale del sughero, Memoria 39, Tempio pausania (sass. Italia), 1974.

[9] Chakali G., Ghalem M., Les insectes ravageurs du chêne liège, Quercus suber L. en Algérie, in: Actes sémin. médit. sur la régénération des forêts de chềne liège, Tabarka-Tunisie, 22-24 octobre 1996, Ann. INRGREF Suppl., 1998, pp. 253-259.

[10] Dagnelie P., Théories et méthodes statistiques, Vol. II, Programmes agronomiques de Gembloux, 1975.

[11] Direction Générale des Forêts, Résultats du premier inventaire forestier national en Tunisie, D.G.F. Tunisie, 1995,

[12] Dhote J.F., Hatsch E., Rittié D., Forme de la tige, tarifs de cubage et ventilation de la production en volume chez le chêne sessile, Ann. For. Sci. 59 (2000) 121-142.

[13] Duvigneaud P., Denaeyer S., Ambroes P., Timpermann J., Recherches sur l'écosystème forêt. Biomasse, Productivité et cycles des polyéléments biogènes dans l'écosystème " chênaies caducifoliées », Essai de phytogéochimie forestière, Inst. Roy. Sci. Nat. de Belgique, Mem. 164, 1971, $101 \mathrm{p}$.

[14] Duvigneaud P., La productivité primaire des écosystèmes terrestres, in: Lamotte M., Bourlière F. (Eds.), Problèmes de Productivité biologique, Masson et Cie Paris, 1977.

[15] Duvigneaud P., Kestemont P., Productivité biologique en Belgique, Gembloux, Duculot, 1977, 617 p.

[16] Ed-Derfoufi F., Gestion et dynamique des nutrients dans des taillis de chêne vert âgés et très jeunes, Thèse $3^{\mathrm{e}}$ cycle USTL Montpellier, $1986,131 \mathrm{p}$.

[17] El Hamrouni A., Végétation forestière et préforestière de la Tunisie: Typologie et éléments pour la gestion, Thèse Université de Provence Aix-Marseille III, 1992, 235 p. 
[18] Esau C., Plant anatomy, 2nd ed., New York, John Wiley and sons, 1977.

[19] Et-Tobi M., Contribution à l'étude de la dynamique et du dépérissement du chêne liège en Mamora (Maroc), Mém. $3^{\mathrm{e}}$ cycle E.N.F.I., Maroc, 1996.

[20] Ezzahri M., Belghazi B., Moulay A., Croissance en épaisseur du liège de reproduction dans les forêts du plateau central : cas de la suberaie de Ait hatem, Annales de l'INRGREF Suppl., 2001, pp. $125-131$.

[21] Favrel P., Priol P., Étude des techniques de boisement en chêne liège : $1^{\text {ers }}$ résultats, in: Actes sémin. médit. sur la régénération des forêts de chêne liège, Tabarka-Tunisie, 22-24 octobre 1996, Ann. INRGREF Suppl., 1998, pp. 102-113.

[22] Ferreira M.C., Carvalho Oliveira A.M., Modelling Cork oak production in Portugal, Agrofor. Syst. 16 (1991) 41-54.

[23] Ferreira A., Lopez F., Pereira H., Caractérisation de la croissance et de la qualité du liège dans une région de production, Ann. For. Sci. 59 (2000) 187-193.

[24] Gallego H.A., Santa Regina I., Rico M., Biomass equations and nutrient distribution for Quercus pyrenaica Wild., Forest, Mésogée 53 (1993) 75-82.

[25] Gonzalez-Adrados J.R., Montéro- Gonzalez G., Ortega-Muela C., Caracterizacion productiva de los alcornocales catalanes, Investig. Agrar. Sist. Recur. For. 2 (1993) 55-69.

[26] Gonzalez-Adrados J.R., Gonzalez-Hernandez F., Variabilidad de los parametros de produccion de corcho in: Pereira H. (Ed.), Cork oak and oak, Centro de Estudos Florestais, Lisboa, 1998, pp. 47-53.

[27] Gower S.T., Grier C.C., Vogt D.J., Vogt K.A., Allometric relations of deciduous (Larix occidentalis) and evergreen conifers (Pinus contorta, and Pseudotsuga menziesii) of the cascade mountains in central Washington, Can. J. For. Res. 17 (1987) 630-634.

[28] Halaoui B., Phytomasse, minéralomasse et productivité des principaux écosystèmes forestiers du Maroc oriental, Actes des Premières Journées de l'Arbre, 1992, pp. 91-93.

[29] Hanchi A., Cycle de l'eau et des éléments biogènes dans un bassin forestier : cas d'une hétraie au Mont Lozère, Thèse Université de Bourgogne, 1994, $232 \mathrm{p}$.

[30] Hasnaoui B., Chênaies du Nord de la Tunisie : Écologie et Régénération, Thèse Université de Provence, Aix-Marseille I, 1992, 202 p.

[31] Haygreen J.G., Bowyer J.L., Forest products and wood science, 1 st ed., Iowa state university press, 1982.

[32] Horisberger D., Étude de la biomasse aérienne du peuplement du Quercus ilex L. de la station du Rouquet, D.E.A. Fac. Sci. Montpellier, 1969.

[33] Laamouri A., Chtourou A., Ben Salem H., Prédiction de la biomasse aérienne d'Acacia cyanophylla Lindl. (Syn. A. saligna (Labill.) H. Wendl) à partir de mensurations dimensionnelles, Ann. For. Sci. 59 (2002) 335-340.

[34] Le Goff N., Ottorini J.M., Root biomass and biomass increment in a beech (Fagus sylvatica L.) stand in North East France, Ann. For. Sci. 58 (2001) 1-13.

[35] Léonardi S., Rapp M., Phytomasse et minéralomasse d'un taillis de chêne vert du massif de l'Etna, Ecol. Mediter. VIII (1982) 125-137.

[36] Léonardi S., Rapp M., Production de phytomasse et utilisation de bioéléments lors de la reconstitution d'un taillis de chêne vert, Acta Oecologia 11 (1990) 819-834.

[37] Léonardi S., Rapp M., Dénes A., Organic matter distribution and fluxes within a holm oak (Quercux ilex L.) stand in the Etna volcano, Vegetatio 99-100 (1992) 219-24.

[38] Léonardi S., Rapp M., Dénes A., Biomasse, minéralomasse, productivité et gestion de certains éléments biogènes dans une forêt de Quercus suber L. en Sicile (Italie), Ecol. Mediter. XVIII (1992) 89-98.

[39] Léonardi S., Rapp M., Failla M., Komaromy E., Organic matter and nutrient cycling within an endemic birch stand in the Etna massif (Sicily): Betula aetnensis Rafin, Vegetatio 111 (1994) 45-59.

[40] Léonardi S., Santa Regina I., Rapp M., Gallego H.A., Rico M., Biomass, litterfall and nutrient content in Castanea sativa coppice stands of southern Europe, Ann. Sci. For. 53 (1996) 1076-1081.

[41] Lossaint P., Rapp M., La forêt méditerranéenne de chênes verts, in: Lamotte et Bourlière, Problèmes d'Écologie, Écosystèmes terrestres, Masson Paris, 1978, pp. 129-185.
[42] Mercurio R., Saba G., Expériences sur la reconstitution de la suberaie en Sardaigne (Italie), in: Actes sémin. médit. sur la régénération des forêts de chêne liège, Tabarka-Tunisie, 22-24 octobre 1996, Ann. INRGREF Suppl., 1998, pp. 35-41.

[43] Merouani H., Acherar M., Istambouli A., Recherches de quelques contraintes biotiques et abiotiques à la régénération naturelle du chêne liège: Quercus suber L., in: Actes sémin. médit. sur la régénération des forêts de chêne liège, Tabarka-Tunisie, 22-24 octobre 1996, Ann. INRGREF Suppl., 1998, pp. 225-243.

[44] Molinas M., Oliva M., Caritat A., Estudio comparativo de la elongación apical y los parámetros foliares en seis parcelas de alcornocal de Girona, Scientia Gerundensis 18 (1992) 61-71.

[45] Montéro G., Grau J.M., Producción de un alcornocal en Santa Coloma de Farners (Girona), Scientia Gerundensis 15 (1989) 131-139.

[46] Montéro G., San Miguel A., Alia R., Estructura y producción de los alcornocales (Quercus suber) del sur de España, Investig. Agrar. Sist. Recur. For. 0 (1991) 69-74

[47] Motte M., Instructions relatives aux travaux de démasclage et de récolte des lièges de reproduction, au transport, au cubage et au triage des lièges sur parc, Service des forêts, $1960,9 \mathrm{p}$.

[48] Natividade J.V., Subericultura. Ministra da Economia, Diriçao General dos servicos Florestais e Aquicolas Lisboa, 1950.

[49] Navar J., Mendez E., Dale V., Estimating stand biomass in the Tamaulipan thornscrub of northeastern Mexico, Ann. For. Sci. 59 (2002) 813-821.

[50] Panshin A.J., De Zeeuw C., Textbook of wood technology, 4th ed., New York, Mc Graw-Hill, 1980.

[51] Pereira H., Chemical composition and variability of cork from Quercus suber L., Wood Sci. Technol. (1988) 211-218.

[52] Ranger J., Marques R., Colin-Belgrand M., Flammang N., Gelhaye D., The dynamics of biomass and nutrient accumulation in a Douglasfir (Pseudotsuga menziesii Franco) stand studied using chronosequence approach, For. Ecol. Manage. 72 (1995) 167-183.

[53] Rapp M., Cycle de la matière organique et des éléments minéraux dans quelques écosystèmes méditerranéens, P.I.B. Recherche Coopérative sur Programme du CNRS $\mathrm{N}^{\circ} 40, \mathrm{CNRS}, 1971$.

[54] Rapp M., Lossaint P., Some aspects of mineral cycling in the garrigue of Southern France, Elsevier Scientific Publishing Company, Amesterdam-Neetherlands, 1981, pp. 289-301.

[55] Rapp M., Ed Derfoufi F., Blanchard A., Productivity and nutrient uptake in a holm oak (Quercus ilex $\mathrm{L}$.) stand during regeneration after clear-cut, Vegetatio 99-100 (1992) 263-272.

[56] Robert B., Caritat A., Bertoni G., Vilar L., Molinas M., Nutrient content and seasonal fluctuations in the leaf component of cork oak (Quercus suber L.) litterfall, Vegetatio 122 (1996) 29-35.

[57] Rognon P., Sécheresse et aridité : leur impact sur la désertification au Maghreb, Cahiers « Sécheresse » 7 (1997) 287-297.

[58] Santa Regina I., Rapp M., Martin A., Gallardo J.F., Nutrient release dynamics in decomposition leaf litter in two Mediterranean deciduous oak species, Ann. Sci. For. 54 (1997) 747-760.

[59] Santelli J., Communication de l'Office National des forêts sur la régénération des forêts de chêne liège varoises, in: Actes sémin. médit. sur la régénération des forêts de chêne liège, Tabarka-Tunisie 22-24 octobre 1996, Ann. INRGREF Suppl., 1998, pp. 48-62.

[60] Saoudi H., Réponse des végétaux aux facteurs de dégradation en Kroumirie (Tunisie), Thèse de Docteur Ingénieur, Univ. AixMarseille III, 1983.

[61] Sebei H., Albouchi A., Rapp M., El Aouni M.H., Évaluation de la biomasse arborée et arbustive dans une séquence de dégradation de la suberaie à Cytise de Kroumirie (Tunisie), Ann. For. Sci. 58 (2001) 175-191.

[62] Yessad Sid A., Le chêne liège et le liège dans les pays de la Méditerranée occidentale, Louvain la Neuve ASBL Forêt wallonne, 2000

[63] Wang J.R., Zhong A.I., Simard S.W., Kimmins J.P., Aboveground biomass and nutrient accumulation in an age sequence of paper birch (Betula papyrifera) in the Interior Cedar Hemlock zone, British Columbia, For. Ecol. Manage. 83 (1996) 27-38.

[64] Woodwell G.M., Whittaker R.H., Primary production in terrestrial communities, Ann. Zool. 8 (1968), 19-30. 